\title{
Regulation of Sufu activity by p66ß and Mycbp provides new insight into vertebrate Hedgehog signaling
}

\author{
Chuwen Lin, ${ }^{1}$ Erica Yao, ${ }^{1}$ Kevin Wang, ${ }^{2}$ Yoko Nozawa, ${ }^{1}$ Hirohito Shimizu, ${ }^{2}$ Jeffrey R. Johnson, ${ }^{3}$ \\ Jau-Nian Chen, ${ }^{2}$ Nevan J. Krogan, ${ }^{3}$ and Pao-Tien Chuang ${ }^{1}$ \\ ${ }^{1}$ Cardiovascular Research Institute, University of California at San Francisco, San Francisco, California 94158, USA; \\ ${ }^{2}$ Department of Molecular, Cell, and Developmental Biology, University of California at Los Angeles, Los Angeles, California \\ 90095, USA; ${ }^{3}$ Department of Cellular and Molecular Pharmacology, University of California at San Francisco, San Francisco, \\ California 94158
}

Control of Gli function by Suppressor of Fused (Sufu), a major negative regulator, is a key step in mammalian Hedgehog $(\mathrm{Hh})$ signaling, but how this is achieved in the nucleus is unknown. We found that Hh signaling results in reduced Sufu protein levels and Sufu dissociation from Gli proteins in the nucleus, highlighting critical functions of Sufu in the nucleus. Through a proteomic approach, we identified several Sufu-interacting proteins, including p66ß (a member of the NuRD [nucleosome remodeling and histone deacetylase] repressor complex) and Mycbp (a Myc-binding protein). p66 $\beta$ negatively and Mycbp positively regulate Hh signaling in cell-based assays and zebrafish. They function downstream from the membrane receptors, Patched and Smoothened, and the primary cilium. Sufu, p66ß, Mycbp, and Gli are also detected on the promoters of Hh targets in a dynamic manner. Our results support a new model of Hh signaling in the nucleus. Sufu recruits p66ß to block Gli-mediated Hh target gene expression. Meanwhile, Mycbp forms a complex with Gli and Sufu without Hh stimulation but remains inactive. Hh pathway activation leads to dissociation of Sufu/p66 $\beta$ from Gli, enabling Mycbp to promote Gli protein activity and $\mathrm{Hh}$ target gene expression. These studies provide novel insight into how Sufu controls Hh signaling in the nucleus.

[Keywords: Sufu; p66ß; Mycbp; Gli; Hh signaling]

Supplemental material is available for this article.

Received July 21, 2014; revised version accepted October 16, 2014.

Hedgehog $(\mathrm{Hh})$ signaling plays a key role in diverse aspects of embryonic development and postnatal physiology (Ingham et al. 2011; Briscoe and Therond 2013; Chen and Jiang 2013; Petrova and Joyner 2014). Perturbation of $\mathrm{Hh}$ signaling is also associated with various types of human cancers (Scales and de Sauvage 2009; Barakat et al. 2010; Bijlsma and Roelink 2010). Elucidating the molecular mechanisms of Hh signaling is essential to our fundamental understanding of developmental processes and disease mechanisms. Hh signal transduction is initiated through binding of the lipidated ligand to the 12-pass transmembrane protein Patched (Ptch1/Ptc1), a process also modulated by Hh coreceptors (Wang et al. 2007; Farzan et al. 2008; Beachy et al. 2010; Ryan and Chiang 2012; Filmus and Capurro 2014). This relieves Ptch1 repression on the seven-pass transmembrane protein Smoothened (Smo) and enables Smo to transduce the Hh signal (Robbins et al. 2012), albeit the precise biochemical

Corresponding author: pao-tien.chuang@ucsf.edu.

Article is online at http://www.genesdev.org/cgi/doi/10.1101/gad.249425.114. functions of Ptch1 and Smo have not been defined. Recent studies suggest that lipid binding to Smo at multiple sites may modulate Hh pathway activity (Myers et al. 2013; Nachtergaele et al. 2013). Three Gli transcription factors (Gli1-3) mediate $\mathrm{Hh}$ responses downstream from Smo in mammals (Hui and Angers 2011; Aberger and Ruiz i Altaba 2014). Gli protein levels and activities are primarily regulated by Suppressor of Fused (Sufu), although protein kinase A (PKA) (Tuson et al. 2011; Niewiadomski et al. 2014) and the kinesin Kif7 (Cheung et al. 2009; Endoh-Yamagami et al. 2009; Liem et al. 2009) also play a key role in controlling Gli protein function. Gli3 undergoes limited proteolysis to generate a transcriptional repressor (GliR) in the absence of $\mathrm{Hh}$ signaling to silence Hh target gene expression (Wang et al. 2000a). Hh

(C) 2014 Lin et al. This article is distributed exclusively by Cold Spring Harbor Laboratory Press for the first six months after the full-issue publication date (see http://genesdev.cshlp.org/site/misc/terms.xhtml). After six months, it is available under a Creative Commons License (Attribution-NonCommercial 4.0 International), as described at http:// creativecommons.org/licenses/by-nc/4.0/. 
signaling not only inhibits the production of Gli repressors but also facilitates the generation of Gli activators (GliA; largely derived from full-length Gli2) to activate Hh target genes, which include Ptch1, Gli1, and Hhip. Gli1 does not undergo limited proteolysis and only functions as a transcriptional activator. Both Gli2 and Gli3 can generate a repressor in the absence of $\mathrm{Hh}$ signaling, but Gli2 proteolysis is inefficient (Pan et al. 2006), and the Gli2R plays a minor role in vivo (Li et al. 2011). Similar to Gli2, full-length Gli3 can function as an activator, but the contribution of Gli3 activator to Hh pathway activity in vivo is insignificant (Bai et al. 2004).

Hh signal transduction in mammals uses the primary cilium (Eggenschwiler and Anderson 2007; Wong and Reiter 2008; Berbari et al. 2009; DeRouen and Oro 2009; Goetz and Anderson 2010; Bay and Caspary 2012; Drummond 2012; Oh and Katsanis 2012; Kim and Dynlacht 2013; Nozawa et al. 2013; Mukhopadhyay and Rohatgi 2014; Nachury 2014), an evolutionarily conserved microtubule-based organelle analogous to the flagella found in single-celled eukaryotes such as Chlamydomonas reinhardtii (green algae). All core components of vertebrate $\mathrm{Hh}$ signaling localize to the primary cilium in a dynamic manner (Corbit et al. 2005; Haycraft et al. 2005; Rohatgi et al. 2007; Chen et al. 2009; Endoh-Yamagami et al. 2009; Kim et al. 2009; Liem et al. 2009). However, correlating ciliary distribution and the movement of Hh pathway components with their biochemical functions in Hh signaling remains a daunting endeavor. A thorough characterization of the dynamic ciliary movement of Hh pathway components coupled with functional studies is required to address this important issue.

Sufu is a major negative regulator of mammalian $\mathrm{Hh}$ signaling. Loss of Sufu in mammals leads to global Hh pathway activation and early embryonic lethality (Cooper et al. 2005; Svard et al. 2006). Sufu thus provides a key tool to understand how $\mathrm{Hh}$ signaling controls target gene activity. It is known that Gli proteins execute their function in the nucleus. Sufu binds Gli proteins (Ding et al. 1999; Kogerman et al. 1999; Pearse et al. 1999; Stone et al. 1999), which display dynamic shuttling between the cytoplasm and nucleus (Kogerman et al. 1999; Kim et al. 2009; Humke et al. 2010). We expect that an essential aspect of Sufu function must reside in its control of Gli activity in the nucleus. Surprisingly, our knowledge of Sufu/Gli activity in the nucleus is very limited. In contrast, Sufu function in the cytoplasm or on the primary cilium is better studied. Sufu has been shown to sequester Gli proteins in the cytoplasm (Ding et al. 1999; Kogerman et al. 1999; Murone et al. 2000; Barnfield et al. 2005), control Gli protein levels (Chen et al. 2009; Jia et al. 2009; Wang et al. 2010), and regulate the production of Gli repressors and activators (Humke et al. 2010; Tukachinsky et al. 2010). Elucidating Sufu's nuclear function would fill a major gap in our mechanistic understanding of Hh signaling.

Canonical Gli-binding sites (GliBSs) have been identified in many $\mathrm{Hh}$ target genes. How various combinations of Gli activators and repressors control Hh target gene expression and confer graded $\mathrm{Hh}$ responses in the nucleus is a major unresolved issue in $\mathrm{Hh}$ signaling (Hui and
Angers 2011; Rabinowitz and Vokes 2012; Falkenstein and Vokes 2014). This task is particularly challenging because different tissues use a unique combination of Gli activator/repressor to produce specific $\mathrm{Hh}$ outputs (i.e., a specific set of Hh targets) necessary for patterning. This point is illustrated by the observation that the Gli2 activator plays a dominant role in neural tube development (Ding et al. 1998; Matise et al. 1998; Bai et al. 2004), while the Gli3R is a key determinant of limb patterning (Bowers et al. 2012; Cao et al. 2013), and a different group of Hh targets is activated accordingly. Moreover, complex interactions between various Gli proteins exist in both neural tube (Liu et al. 2012) and limb patterning (Bowers et al. 2012), and pinpointing the contribution of a given Gli protein is nontrivial.

The basic framework of $\mathrm{Hh}$ signaling is established through the identification and characterization of various Hh pathway components, many of which were initially identified by genetic screens in Drosophila. It has also become clear that while the fundamental aspects of $\mathrm{Hh}$ signaling are conserved across species, divergence in $\mathrm{Hh}$ pathway design has occurred during evolution (Wilson and Chuang 2010). Moreover, mammalian Hh signaling relies on the primary cilium, while most fly cells do not contain a primary cilium (Kornberg 2014; Kuzhandaivel et al. 2014). In this case, $\mathrm{Hh}$ regulators specific to the mammalian $\mathrm{Hh}$ pathway cannot be identified by genetic screens in flies. We envision that proteomic or genomic approaches will offer a powerful tool to uncover these components in order to gain new insight into the mechanism and evolution of Hh signaling (Evangelista et al. 2008; Hillman et al. 2011; Jacob et al. 2011).

Through a proteomic method, we identified two new Hh regulators: $\mathrm{p} 66 \beta$ and Mycbp (Myc-binding protein). Our results show that vertebrate $\mathrm{p} 66 \beta$ is a negative regulator of Hh signaling, while Mycbp is a positive modulator of Hh signaling. Both are used in conjunction with Sufu to control $\mathrm{Hh}$ target gene expression to produce graded $\mathrm{Hh}$ responses. In our model, in the absence of the Hh ligand, Gli (such as Gli2) is bound by Sufu, which recruits p66 $\beta$ to inhibit Gli protein-mediated activation of $\mathrm{Hh}$ targets. Hh pathway activation not only abolishes Sufu/p66 $\beta$ inhibition on Gli but also enables Mycbp to promote Glimediated $\mathrm{Hh}$ target gene expression. These advances represent an important step toward our understanding of how Sufu controls Gli activity in the nucleus.

\section{Results}

Hh signaling reduces Sufu protein levels in the nucleus

To gain insight into Sufu's function in the nucleus, we investigated whether $\mathrm{Hh}$ signaling alters Sufu protein levels in the nucleus as a possible means to modulate its activity. Hh treatment did not lead to obvious changes in total protein levels of Sufu. Since Sufu is more abundant in the cytoplasm than in the nucleus, we reasoned that any changes in nuclear Sufu protein levels in response to $\mathrm{Hh}$ signaling would be obscured by high levels of cytoplasmic Sufu. To test this idea, we fractionated cell lysates to 
separate nuclear and cytoplasmic fractions from $\mathrm{Hh}$ responsive mouse embryonic fibroblasts (MEFs) as previously described (Chen et al. 2009) and determined Sufu protein levels by Western blotting. The purity of the cytoplasmic and nuclear fractions was assessed by cytoplasmic- and nuclear-specific markers (Fig. 1A). We found that Sufu protein levels were reduced by $\sim 60 \%$ in the nuclear but not cytoplasmic fraction in response to $\mathrm{Hh}$ treatment (Fig. 1A). Interestingly, reduction in nuclear Sufu protein levels could be restored by the addition of proteasome inhibitors (such as MG132) (Fig. 1B). This suggests that control of Sufu protein levels in the nucleus is used to regulate Gli protein function.

\section{Hedgehog signaling leads to Sufu dissociation} from Gli2 and Gli3 primarily in the nucleus

We then asked whether Sufu activity is also regulated in the nucleus. Several groups reported reduced Gli3 levels (Humke et al. 2010; Tukachinsky et al. 2010; Wen et al. 2010) and dissociation of Sufu from Gli2 and Gli3 (Humke et al. 2010; Tukachinsky et al. 2010) upon Hh pathway activation. This conclusion was reached using total cell lysate from NIH3T3 cells for immunoprecipitation experiments (Humke et al. 2010; Tukachinsky et al. 2010). We inspected in which subcellular compartment Sufu-Gli2 or Sufu-Gli3 dissociation occurs using lysates from MEFs and NIH3T3 cells. We isolated nuclear and cytoplasmic fractions as described above. We confirmed that Gli3 protein levels were reduced upon Hh stimulation (Supplemental Fig. S1A). However, unlike Gli3, Hh signaling did not lead to reduced Gli2 protein levels (Supplemental Fig. S1A).

We then performed immunoprecipitation using either the cytoplasmic or nuclear fraction to test Sufu-Gli dissociation upon Hh pathway activation. While dissociation of Sufu-Gli3 can be detected to some degree in the cytoplasmic fraction, Sufu-Gli3 dissociation mainly occurs in the nuclear fraction (Fig. 1C; Supplemental Fig. S1B). Similarly, we carried out nuclear-cytoplasmic fractionation and immunoprecipitation to examine the dissociation of Sufu and Gli2 upon Hh stimulation. Dissociation of Sufu-Gli2 was also found largely in the nuclear but not the cytoplasmic fraction in wild-type or Ptch1 ${ }^{-/-}$MEFs (Fig. 1C; Supplemental Figs. S1C, S2; data not shown). We noticed that dissociation between Sufu and Gli2/3 in the nuclear fraction could occur at $30 \mathrm{~min}$ after Hh stimulation (Fig. 1C), within the time frame of reported ciliary localization of $\mathrm{Hh}$ pathway components (Tukachinsky et al. 2010; Wen et al. 2010). Sufu-Gli2/3 dissociation in the nuclear fraction was also detected at 6,12 , or $24 \mathrm{~h}$ after $\mathrm{Hh}$ activation when activation of Glil and reduction in Gli3R levels were apparent (Fig. 1C; Supplemental Fig. S3; data not shown). Together, these results reinforce the notion that important regulation of Gli activities by Sufu resides in the nucleus.

Sufu mutants that fail to sequester Gli proteins in the cytoplasm could still repress Hh reporter activity

To further explore Sufu's function in the nucleus, we tested a few Sufu mutants for their ability to sequester Gli protein and inhibit Gli-mediated Hh responses. We pro-
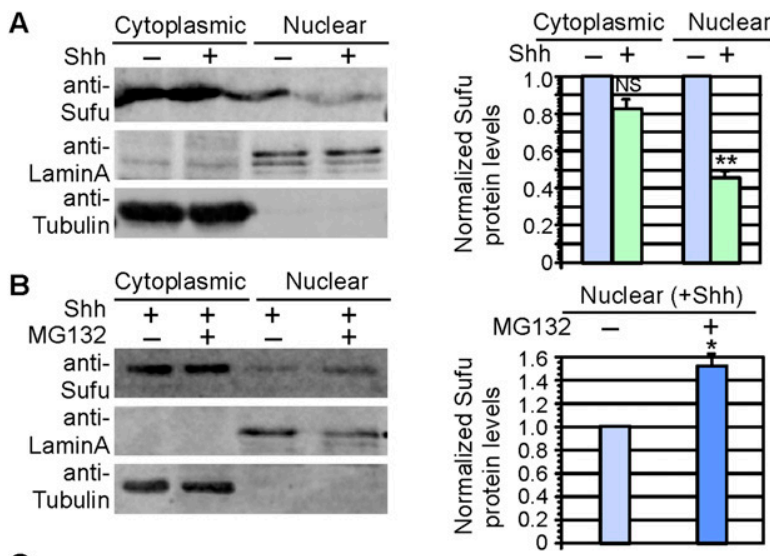

C
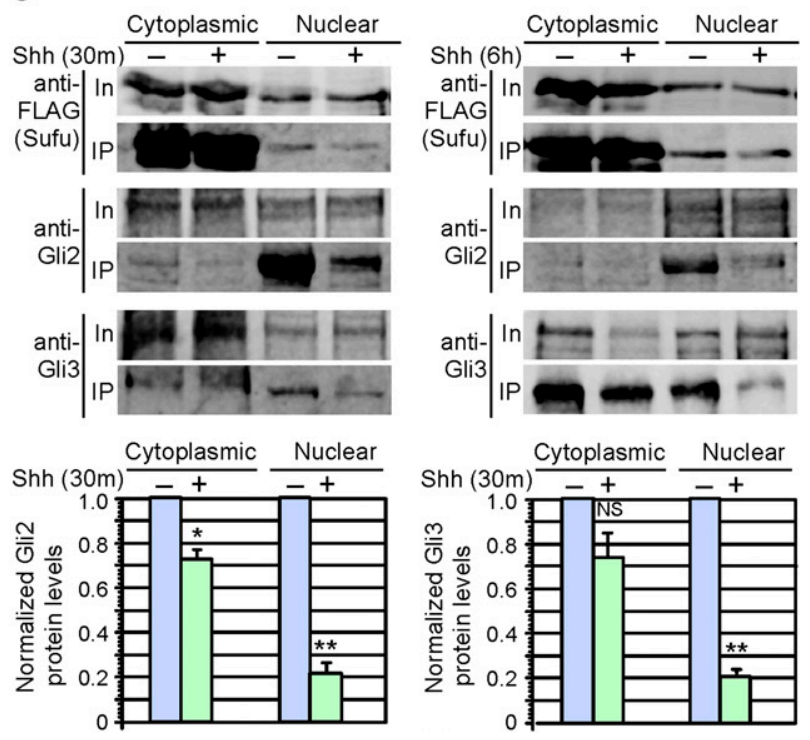

Figure 1. Hedgehog signaling leads to reduced nuclear Sufu protein levels and Sufu dissociation from Gli2 and Gli3 primarily in the nucleus. $(A)$ Western blot analysis and quantification of Sufu protein levels in the nuclear and cytoplasmic fractions derived from MEFs treated with Shh-conditioned medium. Cytoplasmic tubulin and nuclear lamin A were used to assess the purity of cytoplasmic and nuclear fractions. Nuclear Sufu protein levels were reduced by $60 \%$ upon Hh pathway activation, while cytoplasmic Sufu levels were largely unaltered. $(B)$ Western blot analysis and quantification of Sufu protein levels in the nuclear and cytoplasmic fractions in the presence of Shhconditioned medium and proteasome inhibitor MG132. Nuclear Sufu protein levels were notably restored upon MG132 addition. $(C)$ Western blot analysis and quantification of immunoprecipitated Sufu, Gli2, and Gli3 using lysates from the nuclear and cytoplasmic fractions derived from MEFs expressing Flag-tagged Sufu. The amount of coimmunoprecipitated Gli2 and Gli3 by Sufu was significantly reduced in the nuclear fraction but only marginally decreased in the cytoplasmic fraction at indicated time points after Hh stimulation. (In) Input; (IP) immunoprecipitation. $\left({ }^{\star}\right) P<0.05 ;\left(^{\star \star}\right) P<0.01$; (NS) not significant (unpaired Student's $t$-test) $(n=3)$.

duced Sufu ${ }^{\mathrm{N} 100}$ and Sufu ${ }^{\mathrm{N} 212}$ (Barnfield et al. 2005), which retain the first 100 and 212 amino acids of Sufu, respectively. Sufu ${ }^{\mathrm{N} 100}$ and Sufu ${ }^{\mathrm{N} 212}$ failed to sequester Gli proteins in the cytoplasm but were still capable of inhibiting Gli- 
mediated $\mathrm{Hh}$ transcriptional responses in various cell lines, including MEFs, NIH3T3, C2C12, and C3H10T1/2 (Supplemental Figs. S4, S5; data not shown). Readouts of Hh activity were determined by quantitative PCR (qPCR) analysis of Hh target gene expression (such as Gli1) or a standard Hh reporter assay (e.g., 8xGliBS-luc, in which a firefly luciferase [luc] reporter is placed under the control of Hh-responsive element 8xGliBS) (Sasaki et al. 1997). This result confirms and extends previous findings (Barnfield et al. 2005) and highlights a critical function of Sufu in controlling Gli activity in the nucleus that is independent of cytoplasmic sequestering. Taken together, our findings point to an essential role of Sufu in the nucleus in Hh signaling.

\section{A proteomic approach to identify Sufu-interacting proteins uncovers $p 66 \beta$ and $M y c b p$}

In order to further understand the molecular mechanisms by which Sufu regulates Gli protein function, we took a proteomic approach to identify Sufu-interacting proteins other than the three Gli proteins. We generated a stable cell line in MEFs that expresses 3xFlag-Sufu and performed large-scale immunoprecipitation using antiFlag antibodies (Fig. 2A). Endogenous Gli2 and Gli3 were

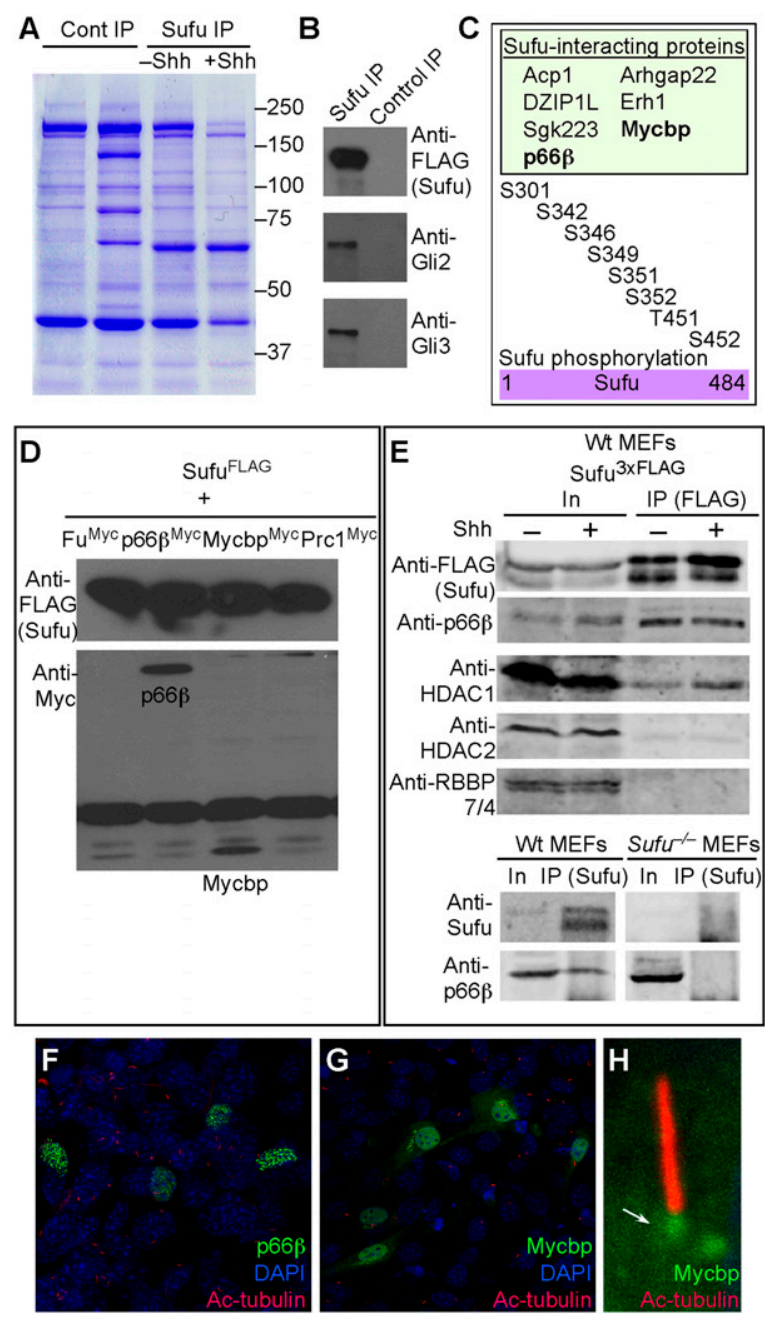

present in the immunoprecipitates, validating our approach (Fig. 2B). Immunoprecipitates were analyzed by mass spectrometry (Jager et al. 2011; Altelaar et al. 2013). We focused on nonstructural proteins with overrepresentations of peptides identified through mass spectrometry. They were good candidates for direct interactions with Sufu. We also treated MEFs expressing 3xFlag-Sufu with Hh-conditioned medium and performed a similar procedure to detect any changes in the levels and composition of proteins that coimmunoprecipitated with Sufu.

We selected a number of Sufu-interacting proteins with overrepresentations of peptides in our proteomic analysis and systematically tested their ability to perturb $\mathrm{Hh}$ signaling through overexpression and knockdown studies. Two of the Sufu-interacting proteins, p $66 \beta$ and Mycbp, showed the most striking and robust effects on Hh signaling and thus are the primary focus of this study (Fig. 2C). p66ß, encoded by Gatad2b (GATA zinc finger

Figure 2. A proteomic approach to identify Sufu-interacting proteins uncovers $\mathrm{p} 66 \beta$ and Mycbp. $(A)$ Coomassie Blue-stained gel of control and Sufu immunoprecipitates treated with mockor Shh-conditioned medium. Distinct bands were detected and were candidates for new Sufu-interacting proteins. Numbers at the right indicate locations of protein size standards. Large-scale immunoprecipitation (IP) and mass spectrometry were performed to identify new Sufu-interacting proteins and Sufu phosphorylation sites. Mass spectrometric analysis was performed directly on immunoprecipitates or specific bands cut out from SDS-PAGE gels. Immunoprecipitation and mass spectrometric analysis were repeated multiple times to eliminate nonspecific Sufu-binding proteins. $(B)$ Western blot analysis of Sufu immunoprecipitates probed with anti-Sufu, anti-Gli2, and Gli3 antibodies. Endogenous Gli2 and Gli3 were detected in Sufu immunoprecipitates (but not in the control), suggesting that a physiologically relevant protein complex was pulled down. (C) Schematic diagram summarizing Sufu-interacting proteins and Sufu phosphorylation sites identified through mass spectrometry analysis. Phosphorylation on S342 and S346 in Sufu is consistent with previous reports (Chen et al. 2011). (D) Western blot analysis of proteins pulled down by Sufu from lysates expressing Sufu and the indicated proteins, which were epitope-tagged. Both p $66 \beta$ and Mycbp physically interacted with Sufu by coimmunoprecipitation. Fu and Prc1 served as negative controls. (E, top panels) Western blot analysis of Sufu immunoprecipitates using lysates derived from MEFs expressing Flagtagged Sufu. Endogenous p $66 \beta$ and HDAC1 were coimmunoprecipitated. In contrast, HDAC2 and RBBP7/4 could not be detected in Sufu immunoprecipitates. (Bottom panels) Western blot analysis of endogenous Sufu immunoprecipitated by an anti-Sufu antibody. p66 $\beta$ was coimmunoprecipitated by Sufu in wild-type MEFs but not in Sufu-deficient MEFs. p66ß/Sufu interaction was not altered by $\mathrm{Hh}$ stimulation (Supplemental Fig. S6). $(F-H)$ Immunofluorescence studies to assess the subcellular distribution of $\mathrm{p} 66 \beta$ and Mycbp. MEFs were transfected or transduced with $\mathrm{p} 66 \beta$ - and Mycbp-expressing constructs. p66 $\beta$ and Mycbp localized to the nucleus (marked by DAPI) of Hh-responsive cells. Cytoplasmic expression of Mycbp was also detected. Acetylated (Ac)-tubulin marks the primary cilium. Interestingly, Mycbp immunoreactivity can also be detected at the base of the cilium (white arrow). 
domain containing $2 B \mid$, is a component of the nucleosome remodeling and histone deacetylase (NuRD) complex (Lai and Wade 2011; Allen et al. 2013). The NuRD complex contains (1) chromodomain helicase DNA-binding protein $3 / 4(\mathrm{CHD} 3 / 4)$, (2) histone deacetylase $1 / 2$ (HDACl/2), (3) methyl cytosine-guanosine (CpG)-binding domain $2 / 3$ (MBD2/3), (4) retinoblastoma-binding proteins $7 / 4$ (RBBP7/4), (5) metastasis-associated 1/2/3 (MTA1/2/3), and $(6) \mathrm{p} 66 \alpha / \beta$. The association between Sufu and p66 $\beta$ offers an opportunity to investigate whether Sufu controls Gli protein activity via the NuRD complex or the Sufu/ p66 $\beta$ complex represents a novel nuclear function of p66 $\beta$ independent of NuRD. Mycbp was originally discovered as a Myc-binding protein capable of stimulating the transcriptional activity of Myc (Taira et al. 1998), and its role in other cellular processes has not been explored.

\section{Both $p 66 \beta$ and Mycbp physically interact with Sufu} and are found in the nucleus

To confirm the physical interaction between Sufu and p66 $3 /$ Mycbp, we expressed epitope-tagged Sufu, p66 $\beta$, and Mycbp in HEK293T cells and performed immunoprecipitation using antibodies against the respective epitopes. We showed that Sufu immunoprecipitates contained $\mathrm{p} 66 \beta$ and vice versa (Fig. 2D; data not shown). In addition, antibodies that pulled down endogenous Sufu in wild-type MEFs also coimmunoprecipitated endogenous p66 $\beta$ (Fig. 2E, bottom panels; Supplemental Fig. S6). These results indicate physical interactions between Sufu and p66 $\beta$ either directly or indirectly through additional proteins. Interestingly, HDAC1 was coimmunoprecipitated with p $66 \beta$ and Sufu, but Sufu did not pull down other known components of the NuRD repressor (Fig. 2E; Supplemental Figs. S7, S8). We also showed that Sufu and Mycbp were coimmunoprecipitated in cultured cells (Fig. 2D). This result indicates that Sufu interacts with Mycbp directly or indirectly.

We examined the subcellular distribution of $\mathrm{p} 66 \beta$ and found that $\mathrm{p} 66 \beta$ protein was largely confined to the nucleus of Hh-responsive cells (Fig. 2F), consistent with its purported nuclear function. Similarly, Mycbp distribution could be found in the nucleus as previously documented (Fig. 2G; Furusawa et al. 2002).

Overexpression of p66 $\beta$ impairs Hh responses in cultured cells, while p $66 \beta$ knockdown results in enhanced Hh pathway activation in cell-based assays

As a first step toward understanding how $\mathrm{p} 66 \beta$ mediates Sufu function in Hh signaling, we overexpressed p $66 \beta$ in Hh-responsive cells (such as NIH3T3 and MEFs) and assessed its effect on Hh pathway activity. Readouts of $\mathrm{Hh}$ activity were determined by a standard Hh reporter assay or transcript levels of $\mathrm{Hh}$ target genes described above. Cotransfection of Gli1 or Gli2 together with $\mathrm{Hh}$ reporters resulted in Hh reporter activation (Fig. 3A). We found that coexpression of $\mathrm{p} 66 \beta$ with Gli1 or Gli2 in this assay severely inhibited Gli-mediated Hh reporter activation or Hh target gene expression, an effect comparable with Sufu-mediated inhibition of Hh pathway activity
(Fig. 3A; Supplemental Fig. S9). Other signaling pathways such as Wnt signaling, assayed by the TOPFlash reporter (Molenaar et al. 1996), were unaffected by p66 $\beta$ overexpression (Fig. 3F), suggesting that p66 $\beta$ does not exert general inhibition of reporter activities. Conversely, shRNAmediated knockdown (Hannon 2003) of $p 66 \beta$ (Supplemental Figs. S10-S12) resulted in enhanced Hh responses. While $p 66 \beta$ knockdown had a modest effect on $\mathrm{Hh}$ reporter activity in the absence of the Hh ligand, reduction in $p 66 \beta$ enhanced $\mathrm{Hh}$ responses upon $\mathrm{Hh}$ stimulation (Fig. 3B; Supplemental Fig. S13). These results are consistent with a negative role of p66 3 in controlling Hh signaling (Supplemental Figs. S14, S15).

Overexpression of Mycbp facilitates Hh responses, while knockdown of Mycbp compromises $\mathrm{Hh}$ responses

We took a similar approach to further understand how Mycbp controls Hh signaling by overexpressing Mycbp in Hh-responsive cells and assessing its effect on Hh pathway activity. Coexpression of Mycbp with Gli1 or Gli2 in this assay significantly promoted Gli-mediated $\mathrm{Hh}$ reporter activation or Hh target gene expression (Fig. 3C; Supplemental Fig. S9). Again, Wnt responses were unaltered by Mycbp overexpression (Fig. 3F). These findings are consistent with a positive role of Mycbp in $\mathrm{Hh}$ pathway activation. Since p $66 \beta$ and Mycbp exert opposite effects on $\mathrm{Hh}$ responses, we anticipate that $\mathrm{p} 66 \beta$ and Mycbp will antagonize each other in controlling $\mathrm{Hh}$ pathway activity. Indeed, when $\mathrm{p} 66 \beta$ and Mycbp were cotransfected in wild-type cells, Mycbp could partially reverse the inhibitory effects of $\mathrm{p} 66 \beta$ on Gli transcriptional activation, likely depending on the Mycbp/p66 $\beta$ ratio (Supplemental Figs. S15, S16).

We also determined the functional consequence of loss of $M y c b p$ through shRNA-mediated knockdown (Hannon 2003) in Hh-responsive cells (Supplemental Fig. S10). We showed that $\mathrm{Hh}$ pathway activation assayed by $\mathrm{Hh}$ reporter activity was severely compromised when Mycbp was knocked down (Fig. 3D). Moreover, Hh target gene expression such as Glil was greatly reduced by Western blotting (Fig. 3E). This further supports a positive role of Mycbp in enhancing $\mathrm{Hh}$ pathway activity.

\section{Expression or knockdown of $p 66 \beta$ and mycbp in zebrafish perturbs Hh signaling, consistent with their respective roles as negative and positive regulators of Hh signaling}

To assess the in vivo function of $p 66 \beta$ and mycbp, we used the zebrafish system as a readout of their in vivo activity. We injected $p 66 \beta$ mRNA into zebrafish embryos and investigated the phenotypic consequences associated with $p 66 \beta$ overexpression. We focused on the developing somites and fin buds, where Hh pathway perturbation leads to well-characterized phenotypes (Lewis et al. 1999; Neumann et al. 1999). We found that $p 66 \beta$ overexpression engendered $U$-shaped somites and reduced fin buds (Fig. 4A,B; Supplemental Fig. S17; data not shown), both 
Lin et al.
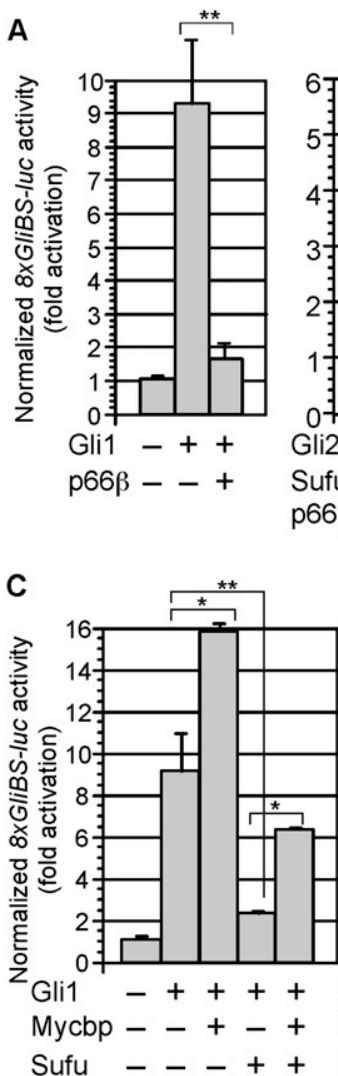

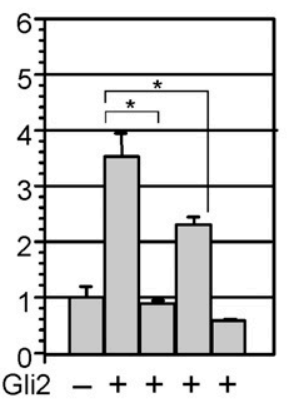

Sufu --+++

$\mathrm{p} 66 \beta--++$

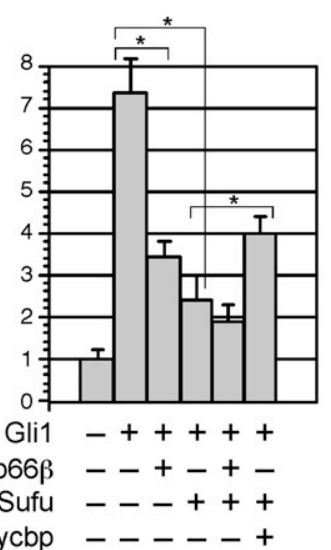

Mycbp
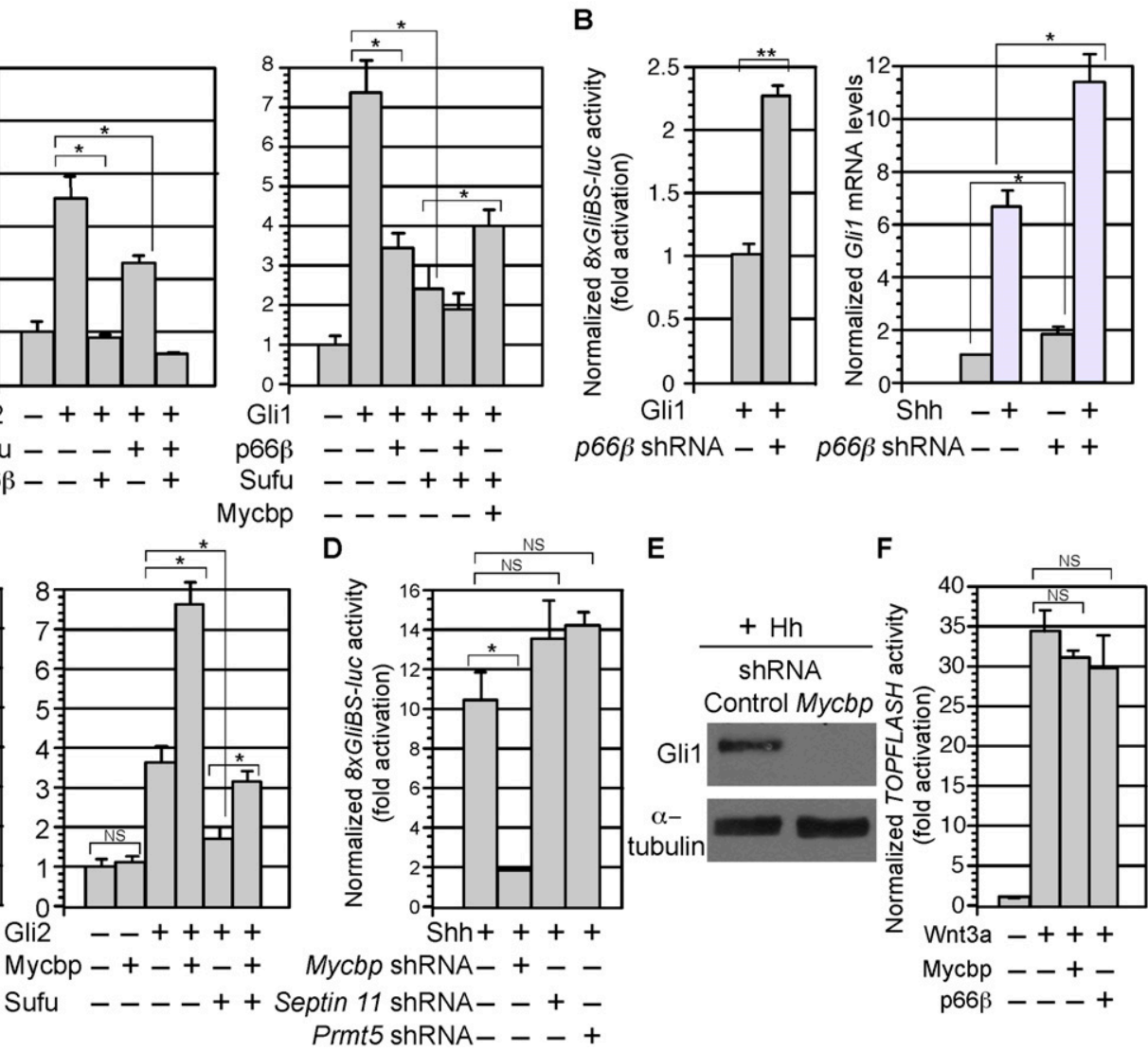

p66 $\beta$ shRNA $-+p 66 \beta$ shRNA -+++

E

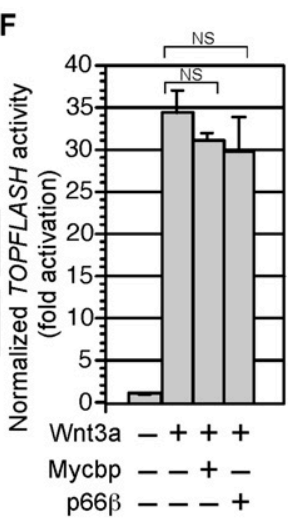

Figure 3. p66 negatively regulates Hh responses, while Mycbp positively regulates Hh signaling in cultured cells. $(A)$ Assessment of p $66 \beta$ activity on Gli-mediated Hh responses using Hh reporter assays in which the $8 x G$ GiBS-luc reporter served as the readouts of Hh signaling. Overexpression of $p 66 \beta$ in NIH3T3 cells abolished Gli-induced Hh reporter activity in a manner similar to Sufu. This supports a negative role of p $66 \beta$ in controlling Hh signaling. (B) shRNA-mediated knockdown of $p 66 \beta$ in MEFs led to increased Hh responses. In this experimental setting, submaximal Hh stimulation was used. $p 66 \beta$ knockdown had no apparent effect on Hh responses when cells received maximal Hh stimulation (Supplemental Fig. S13). Note that knockdown of $p 66 \beta$ in unstimulated cells only caused a modest increase in Hh activity. $(C)$ Assessment of Mycbp activity using Hh reporter assays. Overexpression of $M y c b p$ in NIH3T3 cells potentiated Gli-mediated Hh reporter activity, but Mycbp's positive effects on Hh signaling could be reversed by the presence of Sufu. $(D)$ shRNA-mediated knockdown of Mycbp in MEFs led to decreased Hh target gene expression and compromised Hh responses. Knockdowns of Septin 11 and Prmt5 were used as controls, and no apparent effect on Hh responses was observed. $(E)$ Western blot analysis of Glil protein levels in MEFs in which Mycbp was knocked down by shRNA. Glil was barely induced by Hh stimulation in the absence of Mycbp, consistent with a positive role of Mycbp in Hh responses. (F) p66 3 and Mycbp did not affect Wnt responses using the TOPFlash reporter when NIH3T3 cells were treated with Wnt3a-conditioned medium. Note that Gli1/2 were cotransfected with p $66 \beta$ or Mycbp to assess the effects of $\mathrm{p} 66 \beta / \mathrm{Mycbp}$ on Hh signaling. This is because expression of either a negative or positive component in the Hh pathway (for example, Sufu and Smo, respectively) has little effect on Hh signaling in the steady state (shown in C). In contrast, in the perturbed state (e.g., by expressing Gli), expression of Hh components exerts obvious effects on Hh signaling. Otherwise, expression of p $66 \beta$ and Mycbp, like Sufu and Smo, has no significant effect on Hh signaling in Hh-responsive cells in the steady state. $\left(^{\star}\right) P<0.05 ;\left(^{\star \star}\right) P<0.01$; (NS) not significant (unpaired Student's $t$-test) $(n=3)$.

of which are associated with disruption of Hh signaling. Moreover, Hh target gene expression such as ptch1 was also reduced (or lost) in fin buds when $p 66 \beta$ was overexpressed (Fig. 4C,D,F). Conversely, morpholino (MO)-mediated knockdown of $p 66 \beta$ enhanced $p t c h 1$ expression (Fig. 4C, $\mathrm{E}, \mathrm{H})$. These results support our hypothesis that $\mathrm{p} 66 \beta$ represses $\mathrm{Hh}$ responses.

Similarly, mycbp mRNA or MO was injected into zebrafish embryos, and Hh responses-including phenotypic outcomes and Hh target gene expression-were determined. We showed that overexpression of mycbp in zebrafish embryos resulted in an increase in Hh target gene expression (such as ptch1) in fin buds (Fig. 4C,E,G; Supplemental Fig. S17). In contrast, $p t c h 1$ expression was reduced in mycbp morphants (Fig. 4C,D,I). This is consistent with a positive role of mycbp in Hh signaling. Taken together, these studies provide in vivo evidence to support p66 $\beta$ and Mycbp as new regulators of vertebrate $\mathrm{Hh}$ signaling.

\section{$p 66 \beta$ and Mycbp function downstream}

from Ptch1/Smo/cilia to mediate Sufu activity

Having established the effects of $\mathrm{p} 66 \mathrm{\beta}$ and Mycbp on Hh signaling, we performed similar assays using cell lines de- 
ficient in various Hh pathway components (Chen et al. 2009) to reveal the relationship between $\mathrm{p} 66 \beta$, Mycbp, and other Hh components. We found that $\mathrm{p} 66 \beta$ failed to inhibit Glimediated Hh reporter activity in Sufu-deficient MEFs (Fig. 5; Supplemental Fig. S18), suggesting that p $66 \beta$ activity depends on Sufu. In contrast, p66 $\beta$ was capable of inhibiting Gli-mediated Hh reporter activity in Ptch1or Smo-deficient MEFs (Fig. 5; Supplemental Fig. S18), placing $\mathrm{p} 66 \beta$ downstream from Ptch 1 and Smo in controlling Hh signaling. Furthermore, p $66 \beta$ also inhibited Gli-mediated $\mathrm{Hh}$ reporter activity in Kif3a-deficient MEFs (Fig. 5; Supplemental Fig. S18) in which primary cilia fail to form and Hh signaling cannot be transduced. This indicates that $\mathrm{p} 66 \beta$ activity does not depend on the primary cilium.

Mycbp modestly promoted Gli-mediated Hh reporter activity in Sufu-deficient MEFs (Fig. 5). We speculate that either Sufu or Gli can recruit Mycbp, and Mycbp remains inactive in the absence of the Hh ligand; Mycbp activity is required only when the Hh signal is transduced. Consistent with this, we found that knockdown of Mycbp in Sufudeficient cells blunts Hh target gene expression (Supplemental Fig. S19). In addition, we found that full-length Gli proteins can interact with Mycbp in the absence of Sufu (Supplemental Fig. S20).

Mycbp was capable of stimulating Gli-mediated $\mathrm{Hh}$ reporter activity in Ptch1- or Smo-deficient MEFs (Fig. 5), suggesting that Mycbp functions downstream from Ptch1 and Smo. In addition, Mycbp also potentiated Gli-mediated Hh reporter activity in Kif3a-deficient MEFs (Fig. 5), indicating that Mycbp activity is independent of the primary cilium. Finally, overexpression of p $66 \beta$ and Mycbp does not influence ciliary localization of Gli2 (data not shown). Together, these findings show that $\mathrm{p} 66 \beta$ and Mycbp function downstream from Ptch1/Smo/cilia and likely at a similar step in the Hh pathway in mediating Sufu's nuclear activity.
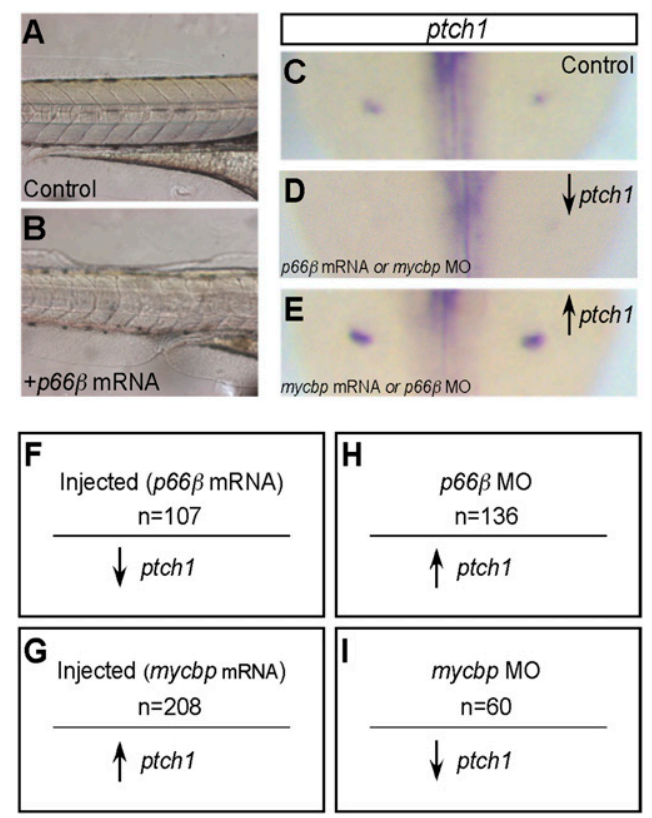

Protein complex formation occurs between Sufu, Gli, and $p 66 \beta / M y c b p$

Since Sufu interacts with both $p 66 \beta$ and Gli, we surmise that Sufu, p66 $\beta$, and Gli may form a multicomponent protein complex, and Sufu bridges the interactions between p66 $\beta$ and Gli. Consistent with this model, Gli2 immunoprecipitates from MEFs expressing 3xFlag-Gli2 contained both Sufu and p66 (Fig. 6A; Supplemental Fig. S21), while coimmunoprecipitation of $\mathrm{p} 66 \beta-\mathrm{Gli2}$ failed to occur in Sufu ${ }^{-/-}$MEFs that express 3xFlag-Gli2 (Fig. 6B). Similar interactions between full-length Gli3, Sufu, and p66 $\beta$ were obtained in MEFs expressing 3xFlag-Gli3 (Fig. $6 \mathrm{~A}, \mathrm{~B} ;$ Supplemental Fig. S21). This suggests that Sufu can recruit $\mathrm{p} 66 \beta$ to control Gli activity. Using a parallel approach, we found that Mycbp bound to full-length

Figure 4. Expression of $\mathrm{p} 66 \beta$ in zebrafish inhibits Hh signaling while expression of mycbp enhances Hh signaling. $(A)$ Lateral view of chevron-shaped somites in wild-type zebrafish embryos at $3.5 \mathrm{~d}$ post-fertilization. $(B)$ Injection of $p 66 \beta$ mRNA resulted in U-shaped somites, consistent with disruption of Hh signaling. $(C-E)$ Whole-mount in situ hybridization to ptch1 in wild-type zebrafish embryos and embryos injected with either $p 66 \beta /$ mycbp mRNA or $p 66 \beta / m y c b p$ MO. Embryos were collected at $36 \mathrm{~h}$ post-fertilization (hpf). ptch1 expression in the fin buds was significantly reduced in embryos injected with $p 66 \beta$ mRNA or mycbp MO. In contrast, injection of mycbp mRNA or $p 66 \beta$ MO increased $p t c h 1$ expression. Since $p t c h 1$ expression is similar in embryos receiving $p 66 \beta$ mRNA/mycbp MO (ptch1 repression) or mycbp mRNA/p66 $\beta$ MO (ptch1 up-regulation), only one representative image of each is shown to indicate changes in ptch1 expression. $(F-I)$ Classification of ptch1 expression levels in fin buds by in situ hybridization in wild-type embryos and embryos injected with $p 66 \beta / m y c b p$ mRNA or $p 66 \beta /$ mycbp MO as indicated. The number $(n)$ of embryos analyzed is specified. Some variation in ptch1 expression levels in the uninjected embryos was noted. Compared with uninjected controls, a higher percentage of embryos injected with $p 66 \beta$ mRNA exhibited weak ptch1 expression $(F)$, while $p t c h 1$ expression was enhanced in a significant proportion of $p 66 \beta$ morphants $(G)$. Overexpression of $p 66 \beta(n=107)$ resulted in, on average, $27.2 \%$ fewer ptch1-expressing embryos than uninjected control embryos $(n=191)$. Conversely, there were $35.0 \%$ more ptch1expressing embryos in the $p 66 \beta$ MO-injected group $(n=136)$ compared with the uninjected control group $(n=65)$. $P$-value $<$ 0.0001 (two-proportion $z$-test) for injection with either $p 66 \beta$ mRNA or $p 66 \beta \mathrm{MO}$. The trend of $p t c h 1$ expression was reversed in embryos injected with mycbp mRNA or mycbp MO. A large fraction of embryos expressing exogenous mycbp mRNA showed strong ptch1 expression $(H)$, and mycbp knockdown significantly reduced ptch1 expression $(I)$. Overexpressing mycbp $(n=208)$ resulted in $33.9 \%$ more embryos expressing ptch1 than uninjected control embryos $(n=303)$. In contrast, knockdown of mycbp $(n=60)$ led to $76.7 \%$ fewer embryos expressing ptch1 than uninjected control embryos $(n=46)$. $P$ value $<0.0001$ (two-proportion $z$-test) for injection with either mycbp mRNA or mycbp MO. Off-target effects of MO-mediated knockdown pose a potential concern (Schulte-Merker and Stainier 2014), and future investigations using genome-editing technology (Harrison et al. 2014), such as CRISPR/Cas or TALENs, will complement these studies. 

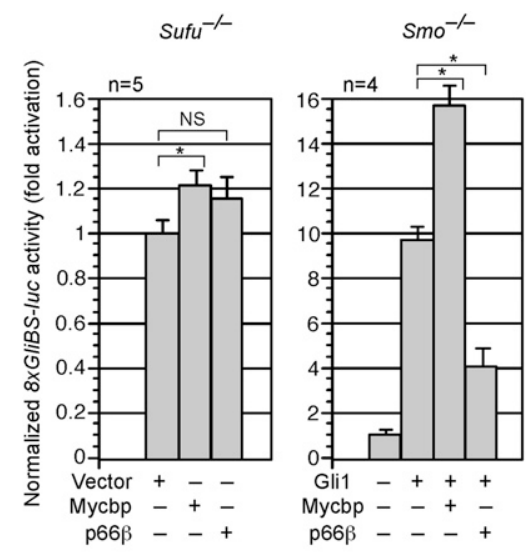

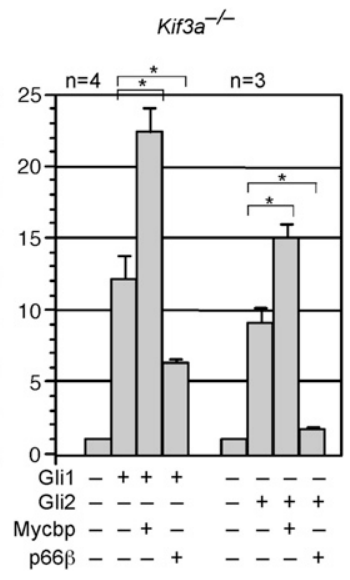

Figure 5. $p 66 \beta$ and Mycbp function downstream from Ptch1, Smo, and the primary cilium to mediate Sufu activity. Placement of p66 $\beta$ and Mycbp in the Hh pathway using cell-based assays. Expression of p $66 \beta$ failed to affect $\mathrm{Hh}$ responses assessed by the $8 \times$ GliBSluc reporter assays in Sufu-deficient MEFs. Sufu-deficient MEFs exhibit strong (albeit submaximal) Hh pathway activation in the absence of Hh ligands. This is consistent with a model in which the function of $\mathrm{p} 66 \beta$ depends on Sufu. Mycbp modestly promoted Gli-mediated Hh reporter activity in Sufudeficient MEFs. In contrast, p66 $\beta$ inhibited, while Mycbp potentiated, Gli-mediated $\mathrm{Hh}$ responses in Smo, Ptch1, or Kif3a mutant MEF lines in a pattern similar to that observed in wild-type MEFs. This suggests that p $66 \beta$ and Mycbp function downstream from membrane receptors Ptch1 and Smo and independently of the primary cilium. Note that Ptch1-deficient MEFs exhibit strong or even maximal Hh pathway activation in the absence of Hh ligands; Hh reporters can still be activated in Ptch1-deficient MEFs if Hh pathway components are exogenously overexpressed. By comparison, Smo-deficient or Kif3adeficient MEFs are defective in Hh signal reception and transduction, and 8xGliBS-luc reporters can be activated only by exogenous nuclear Gli proteins in these cell lines. Gli1/2 were cotransfected with p66 $\beta$ or Mycbp into Smo- and Kif3a-deficient MEFs to assess the effects of p66ß/Mycbp on Hh signaling. Otherwise, expression of $\mathrm{p} 66 \beta$ and Mycbp has no significant effect on Hh signaling in the uninduced state as discussed in the legend for Figure 3. $\left(^{\star}\right) P<0.05$; (NS) not significant (unpaired Student's $t$-test) ( $n$ number is indicated). Similar results and conclusions were obtained by assessing the expression of endogenous Hh targets as the readouts of Hh signaling (Supplemental Fig. S18).

Gli2 or Gli3 protein in addition to Sufu (Fig. 6C), providing evidence to support the presence of a Sufu/Mycbp/Gli protein complex. It is interesting to note that no significant interaction was detected between Gli repressors and p66 $\beta$ or Mycbp (Supplemental Fig. S21).

Since p $66 \beta$ protein levels do not appear to alter upon Hh stimulation (Fig. 2E), we envision two possible scenarios for $\mathrm{p} 66 \beta$ action. Hh pathway activation could lead to dissociation of the Sufu/p $66 \beta$ protein complex from Gli, thus terminating the inhibitory effects on Gli proteins. Alternatively, Hh signaling may result in disruption of the Sufu/p66 $\beta$ protein complex. To distinguish between these possibilities, we performed coimmunoprecipitation experiments using cell lysates from Hh-responsive cells expressing p $66 \beta$, Sufu, and Gli. Cells were also treated with Hh-conditioned medium or agonists to activate the $\mathrm{Hh}$ pathway. We found that $\mathrm{p} 66 \beta$ coimmunoprecipitated with Sufu without Hh stimulation, and p66 $3 /$ Sufu interaction was not altered by Hh stimulation (Fig. 2E; Supplemental Fig. S6). Moreover, p66 $\beta$ dissociates from Gli2 (but not Sufu) upon Hh pathway activation in coimmunoprecipitation experiments (Supplemental Fig. S22). In conjunction with our demonstration of Sufu dissociation from Gli2/3 in the nucleus upon Hh activation (Fig. 1C; Supplemental Fig. S1), these results suggest that Sufu mediates the interaction between $\mathrm{p} 66 \beta$ and Gli, and the entire Sufu/p66 $\beta$ protein complex is released from Gli when the Hh pathway is activated.

\section{Sufu and p663 occupy the promoter of Hh-responsive genes in a Hh-dependent manner}

To further understand how Sufu and p $66 \beta$ control Hh signaling, we asked whether Sufu and p $66 \beta$ could occupy the promoter of Hh-responsive genes, many of which contain canonical GliBSs. This would provide mechanistic insight into how p66 $\beta$ modulates Sufu activity in controlling Hh signaling.

We first assessed the ability of Sufu and Gli proteins to recognize the GliBS. We used double-stranded oligonucleotides that contain a GliBS (denoted Oligo ${ }^{\text {GliBS}}$ ) (Pan et al. 2006) for immunoprecipitation. Oligonucleotides that contain the mutant GliBS (Oligo ${ }^{\Delta \mathrm{GliBS}}$ ) were used as a control. We showed that endogenous Sufu, Gli2, Gli3, and Gli3R were immunoprecipitated by Oligo ${ }^{\text {GliBS }}$ but not Oligo ${ }^{\Delta \mathrm{GliBS}}$ in the absence of Hh ligand stimulation (Fig. 6D). This provides strong evidence to support the notion that a Sufu-Gli protein complex can interact with the GliBS. Interestingly, Sufu failed to be immunoprecipitated by Oligo ${ }^{\text {GliBS }}$ in $\mathrm{Gli2}^{-/-}$; $\mathrm{Gli}^{-/-}$MEFs (Fig. 6E; Zeng et al. 2010) in which all three Gli proteins are absent, since Gli1 expression relies on active Hh signaling via Gli2/3 (Bai et al. 2004). This is consistent with the idea that Sufu association with the GliBS depends on Gli proteins. Hh stimulation led to decreased association between Sufu and the GliBS as well as between Gli3R and the GliBS, while the amount of Gli2 and full-length Gli3 pulled down by the GliBS was significantly increased (Fig. 6D). This supports the notion of Sufu dissociation from Gli proteins upon Hh pathway activation. Given the interactions between Sufu, p66 $\beta$, and Gli, these findings suggest that Sufu can bridge $p 66 \beta$ and Gli interactions on Hh-responsive promoters.

To further test this idea, we performed chromatin immunoprecipitation (ChIP) assays (Collas 2011) on Glil, Gli2, Sufu, and p663. Epitope (e.g., 3xFlag)-tagged Gli1, Gli2, Sufu, and p66 3 were expressed in Hh-responsive cells, and ChIP analysis was performed on known Hh 
target genes (e.g., Ptch1, Gli1, and Hhip) (Goodrich et al. 1996; Lee et al. 1997; Chuang and McMahon 1999|, the promoter of which contains canonical GliBSs. We showed that Gli1, Gli2, and Sufu proteins were enriched on the promoters of Hh-responsive genes (Fig. 6F; Supplemental Fig. S23), consistent with results from immunoprecipitation studies using GliBS oligonucleotides. Importantly, p66 $\beta$ was also enriched on Hh-responsive promoters (Fig. 6F; Supplemental Fig. S23). This was the first demonstration of the presence of Sufu and $p 66 \beta$ on the promoter of Hhresponsive genes. $\mathrm{p} 66 \beta^{\prime} \mathrm{s}$ chromatin association was abolished in Sufu-deficient MEFs (Supplemental Fig. S23), again consistent with the model in which Sufu recruits p66 $\beta$ to Hh target gene promoters. These results suggest that Sufu, Gli, and p66 3 are present at the GliBSs of Hhresponsive genes prior to Hh pathway activation. In this way, Sufu recruits p66 $\beta$ to inhibit Gli-mediated Hh gene expression. This model predicts that Hh pathway activation

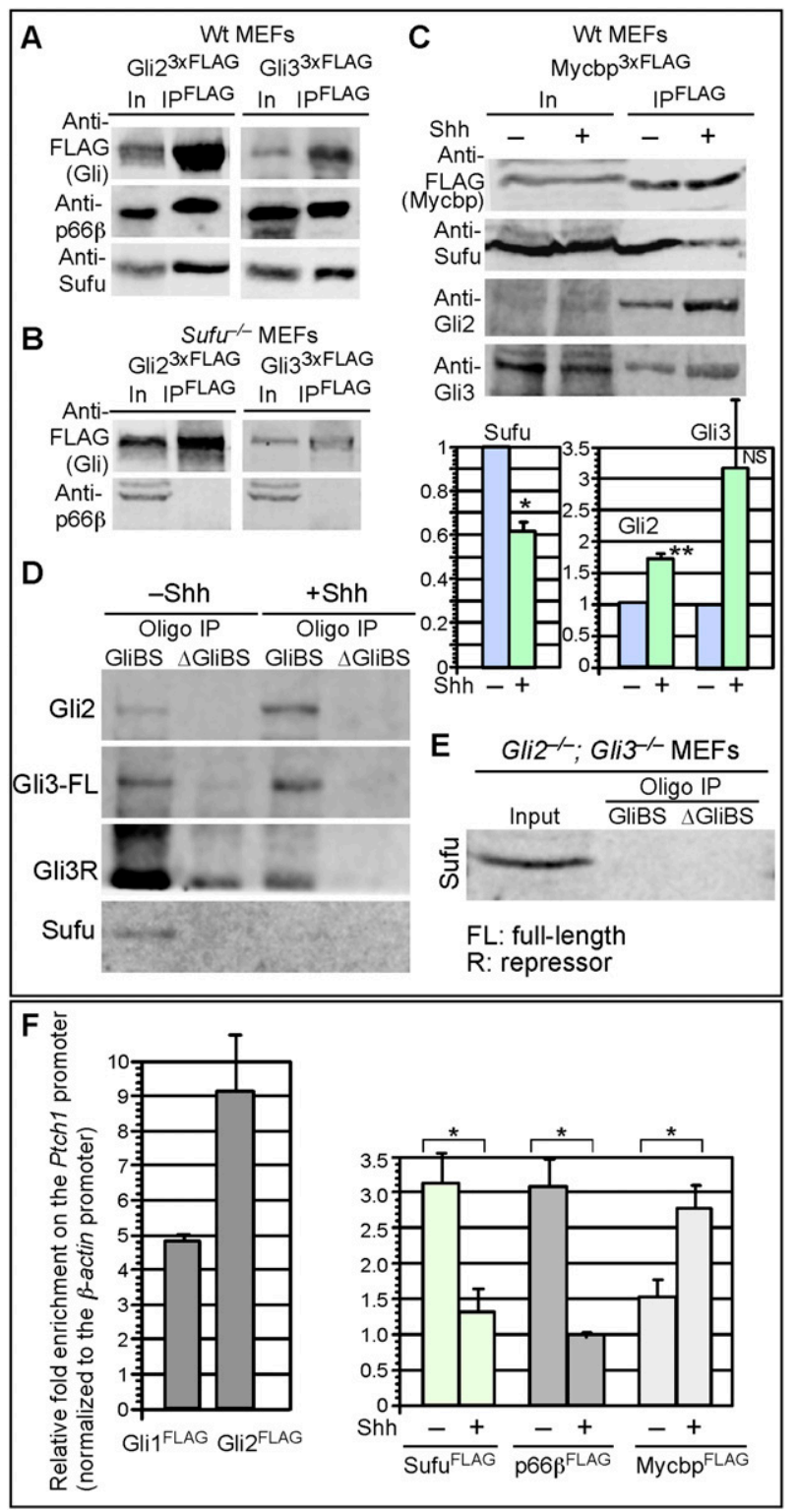

would relieve the inhibition of Sufu/p66 $\beta$ on Gli proteins on $\mathrm{Hh}$ target gene promoters. Indeed, ChIP analysis revealed diminished association of both Sufu and p66 $\beta$ with $\mathrm{Hh}$ promoters upon Hh stimulation (Fig. 6F; Supplemental Fig. S23). These studies yield novel insights into the dynamic interactions of Gli, Sufu, and p66ß on the promoter in the process of Hh pathway activation.

\section{Enhanced interactions between Mycbp and Gli during Hh signal transduction}

Our studies show that $\mathrm{p} 66 \beta$ inhibits Hh responses, while Mycbp promotes Hh responses. Since both p66 $\beta$ and Mycbp interact with Sufu, this led to our hypothesis that Mycbp can potentiate Gli activity once Hh pathway activation removes Sufu/p66 $\beta$ from Gli proteins. To further test this idea, we studied the interaction between Mycbp, Sufu, and Gli proteins during Hh pathway activation. We found that Mycbp dissociated from Sufu upon Hh treatment, while Mycbp interaction with full-length Gli2 and Gli3 was enhanced upon Hh pathway activation (Fig. 6C).

Figure 6. Complex formation between Sufu, p66 $\beta$, Mycbp, and Gli proteins and their dynamic interactions on the promoters of Hh-responsive genes. (A) Western blot analysis of immunoprecipitates using lysates from MEFs expressing Flag-tagged Gli2 or Gli3. Endogenous p $66 \beta$ and Sufu were coimmunoprecipitated, consistent with the formation of a Sufu/p $66 \beta /$ Gli protein complex. $(B)$ Western blot analysis of immunoprecipitates using lysates from Sufu-deficient MEFs expressing Flag-tagged Gli2 or Gli3. Endogenous p $66 \beta$ failed to be coimmunoprecipitated, suggesting that Sufu bridges the interaction between $\mathrm{p} 66 \beta$ and Gli protein. $(C)$ Western blot analysis of immunoprecipitates using lysates from MEFs expressing Flag-tagged Mycbp. Endogenous Sufu, Gli2, and Gli3 were coimmunoprecipitated, implying the production of a Sufu/Mycbp/Gli protein complex. Moreover, Mycbp dissociated from Sufu upon Hh treatment, while Mycbp interaction with full-length Gli2 and Gli3 was enhanced upon Hh pathway activation. $\left(^{\star}\right) P<0.05$; $\left(^{\star \star}\right) P<0.01$; (NS) not significant (unpaired Student's $t$-test) $(n=3)$. Mycbp/ Gli3 interaction was increased upon Hh stimulation, but variations in immunoprecipitations affected the calculated statistical value. (D) Immunoprecipitation of Gli2, Gli3, and Sufu using oligonucleotides that contain a canonical GliBS or control oligonucleotides in which the GliBS is mutated (denoted as $\triangle$ GliBS). Sufu, full-length (FL) Gli2, full-length Gli3, and Gli3R were immunoprecipitated by a GliBS without Hh stimulation but not by the control $\Delta \mathrm{GliBS}$. Hh stimulation led to an increased binding of Gli2 and Gli3 to the GliBS, while association of Sufu or Gli3R with the GliBS was weakened. (E) Sufu failed to be immunoprecipitated by the GliBS in $\mathrm{Gli2}^{-/-}$; $\mathrm{Gli3}^{-/-}$ MEFs, indicating that Sufu binding to the GliBS is dependent on Gli proteins. (F) ChIP analysis of Gli1, Gli2, Sufu, p66 $\beta$, and Mycbp on the promoter of Hh-responsive genes such as Ptch1. Gli1, Gli2, Sufu, and p $66 \beta$ proteins were enriched on the Ptch1 promoter by ChIP. Hh treatment led to the reduced presence of Sufu and $\mathrm{p} 66 \beta$ on the Ptch1 promoter, while binding of Mycbp to the Ptch1 promoter was enhanced. Interestingly, enhanced binding of Mycbp to Hh target gene promoters was abolished in $\mathrm{Gli}^{-/-}$; $\mathrm{Gli3}^{-1-}$ MEFs (Supplemental Fig. S24), suggesting that the promoter occupancy of Mycbp is dependent on Gli proteins. $\left({ }^{\star}\right) P<$ 0.05 (unpaired Student's $t$-test) $(n=3)$. 
We also performed ChIP analysis of Mycbp on the promoters of Hh target genes. In the presence of Hh signaling, Mycbp was enriched at the Hh promoters (Fig. 6F; Supplemental Fig. S23), and enhanced Mycbp binding was abolished in $\mathrm{Gli2}^{-/-}$; Gli3 ${ }^{-/-}$MEFs (Supplemental Fig. S24). This is in contrast to reduced binding of Sufu or p $66 \beta$ to Hh promoters by Hh pathway activation (Fig. 6F; Supplemental Fig. 23). These findings suggest that Hh signaling leads to dissociation of Sufu/p66 $\beta$ from Gli, allowing Mycbp/Gli to activate Hh target gene expression.

\section{Discussion}

Our studies on Sufu-interacting proteins p $66 \beta$ and Mycbp offer novel insight into the regulation of $\mathrm{Hh}$ target gene expression by Sufu and Gli in the nucleus. In particular, p66 $\beta$ and Mycbp modulate the process of how Sufu controls Gli protein function in the nucleus (Fig. 7). Our investigation thus provides a new framework for understanding Hh target gene expression and the production of graded $\mathrm{Hh}$ responses in diverse tissues, a key unresolved issue in Hh signaling.

\section{The multiple roles of Sufu in controlling Gli protein functions}

Extensive studies on Sufu uncovered its role in regulating Gli activities at several subcellular locales and multiple levels. Sufu can sequester Gli proteins (Ding et al. 1999; Kogerman et al. 1999; Murone et al. 2000; Barnfield et al. 2005), regulate Gli protein levels (Chen et al. 2009; Jia et al. 2009; Wang et al. 2010), promote the production of Gli repressors, and inhibit the generation of Gli activators (Humke et al. 2010; Tukachinsky et al. 2010). Physical sequestration of Gli proteins by Sufu in the cytoplasm is traditionally viewed as a major mechanism of inhibiting Gli activities. Recently, Sufu was shown to control Gli protein levels through its antagonistic effects on Spop- or Numb-mediated Gli ubiquitination and degradation /Chen et al. 2009; Wang et al. 2010; Di Marcotullio et al. 2011; Lin et al. 2014). In this way, Sufu preserves a pool of Gli protein that would be available to activate $\mathrm{Hh}$ target gene expression once the Hh signal is transduced. Interestingly, SufuGli association gained a new level of complexity in light of the proposal in which Sufu inhibits Gli activity on the primary cilium (Humke et al. 2010; Tukachinsky et al. 2010). In this model, Hh signaling leads to Sufu-Gli dissociation on the cilium, resulting in Gli activation and the production of a labile form of Gli, which is proposed to be derived from phosphorylated Gli proteins (Humke et al. 2010; Niewiadomski et al. 2014).

The relative contributions of various effects of Sufu to Gli protein activities have not been accurately assessed. In fact, it has not been unambiguously demonstrated where Sufu controls Gli activities. This is largely due to technical difficulty in selective inactivation of endogenous Sufu in a particular subcellular compartment and determining its functional consequence. The notion of a labile active form of Gli is consistent with prior work in Drosophila on Ci, the Gli homolog (Ohlmeyer and Kalderon 1998). However, it
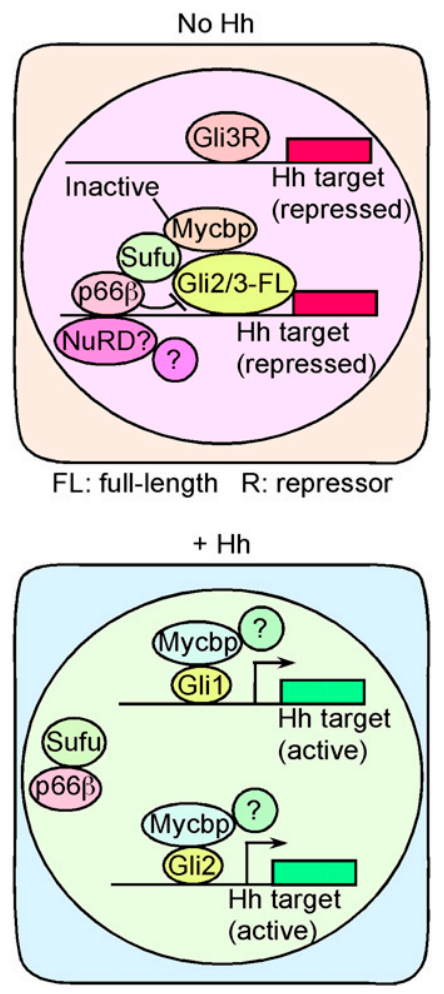

Figure 7. A model of how a p66 $3 /$ Sufu complex inhibits Gli transcriptional activity and a Mycbp/Gli complex enhances Gli transcriptional activity in the nucleus. In the absence of $\mathrm{Hh}$ signaling, Sufu-bound Gli (such as Gli2) is inactive, since Sufu recruits p66 (and other proteins) to block Gli-mediated Hh target gene expression. Hh pathway activation leads to dissociation of Sufu/p66 $\beta$ from Gli, releasing Gli inhibition and priming Gli protein for activating Hh targets. p66 $\beta$ may recruit components of the NuRD repressor complex or other players to inhibit Gli-mediated Hh responses. We propose that Mycbp is recruited to the Sufu/Gli protein complex in the absence of $\mathrm{Hh}$ signaling but remains inactive. How Mycbp is inactivated is not known. Upon Hh pathway activation, Sufu/p $66 \beta$ dissociation from Gli (likely Gli1/2) enhances Mycbp/Gli interaction and enables Mycbp to promote Gli protein activity and Hh target gene expression. The molecular mechanism by which Mycbp enhances $\mathrm{Hh}$ responses is unclear. Post-translational modifications of Sufu, Gli, p66 3 , and Mycbp could influence their interactions and confer important properties of $\mathrm{Hh}$ responses. It is also possible that other activators/repressors also control nuclear Gli activity and exhibit functional redundancy with p66 $\beta /$ Mycbp. (R) Repressor; (FL) full-length.

should be noted that the addition of Hh ligands does not lead to reduced Gli2 protein levels (Supplemental Fig. S1; Kim et al. 2009). Identifying Sufu mutants that affect unique aspects of Gli protein activities will provide critical tools to reveal the contributions of various Sufu/Gli interactions to Hh signaling.

While Sufu-Gli dissociation can be detected to some extent in the cytoplasm, the main site of Sufu-Gli dissociation seems to reside in the nucleus. The extent of dissociation between Gli2/3 and Sufu using nuclear fractions seems to be comparable with that using whole-cell lysates (Humke et al. 2010). We speculate that changes in 
Sufu and Gli distribution upon Hh activation are dynamic and quantitative, not "all or none." In this model, a significant fraction of Sufu and Gli still associate with each other in the cytoplasm, perhaps as a reservoir. Consistent with this idea, Sufu and Gli accumulate in the primary cilium upon Hh stimulation, and, in fact, $\mathrm{Sufu} /$ Gli could still be detected in the cilium $24 \mathrm{~h}$ after Hh treatment.

Perhaps Sufu-Gli dissociation in different subcellular locales serves to execute Sufu's multiple functions in $\mathrm{Hh}$ signaling. For instance, Sufu-Gli dissociation in the cytoplasm/cilium may blunt the generation of Gli repressors and lead to the production of Gli activators, while nuclear Sufu-Gli dissociation could free Gli proteins of their transcriptional repressors.

X-ray crystallographic structures of Sufu in a complex with a short Gli peptide (containing the Sufu-binding SYGH motif) revealed a clamp-like structure of Sufu that interacts with the Gli peptide (Cherry et al. 2013; Zhang et al. 2013). This raised the possibility that both the $\mathrm{N}$-terminal and C-terminal domains of Sufu are required for Gli binding. However, previous studies have reported physical interactions between Sufu truncation mutants (e.g., the N-terminal fragment of Sufu) and Gli proteins (Merchant et al. 2004). Moreover, Gli2 (ASYGH) can still bind Sufu (Santos and Reiter 2014), suggesting the presence of multiple Sufu-binding domains in Gli2. It is possible that Sufu in a multiprotein complex in its native environment would display complex dynamic behaviors, and multiple domains of Sufu and Gli can interact with each other independently. In addition, Gli2 $(\Delta \mathrm{SYGH})$ is not sequestered in the cytoplasm and instead is enriched in the nucleus but does not lead to Hh pathway hyperactivation (Santos and Reiter 2014). This suggests that Sufu can inhibit Gli2 ( $\triangle \mathrm{SYGH}$ ) activity in the nucleus, although it does not sequester Gli2 ( $\triangle \mathrm{SYGH})$ in the cytoplasm. This is consistent with our finding that the sequestering function of Sufu can be separated from its ability to inhibit Gli-dependent transcription as revealed by the N-terminal fragments of Sufu $\left(\mathrm{Sufu}^{\mathrm{N} 100}\right.$ and Sufu $\left.{ }^{\mathrm{N} 212}\right)$. While Sufu ${ }^{\mathrm{N} 100}$ and Sufu ${ }^{\mathrm{N} 212}$ can bind Gli proteins, albeit less strongly than Sufu (Supplemental Fig. S5), and are potent in inhibiting Hh responses in cell-based assays, it is possible that overexpression augmented their inhibitory effects. The function of Sufu ${ }^{\mathrm{N} 100}$ and Sufu ${ }^{\mathrm{N} 212}$ would need to be assessed by introducing these mutants in vivo through gene targeting or genome editing.

Whether Sufu plays a key role in controlling Gli protein functions on Hh-responsive genes in the nucleus has not been extensively studied (Cheng and Bishop 2002). In this regard, our study represents a major step toward understanding Sufu's nuclear function. While we focused on $\mathrm{p} 66 \beta$ and Mycbp in this study, other Sufu-interacting proteins discovered from our proteomic approach could shed light on Sufu function.

\section{Regulation of Gli activity by Sufu and $p 66 \beta$}

We showed that $\mathrm{p} 66 \beta$ negatively regulates Hh signaling in cell-based assays and zebrafish, and p $66 \beta$ relies on Sufu to mediate its inhibitory action on Hh signaling. $\mathrm{p} 66 \mathrm{\beta}$ is a member of the NuRD repressor complex (Lai and Wade 2011; Allen et al. 2013), suggesting that p66 $\beta$ functions in the nucleus. This is further supported by the nuclear distribution of $\mathrm{p} 66 \beta$, its presence on Hh promoters, and the independence of $\mathrm{p} 66 \beta$ activity on Ptch1/Smo and the primary cilium.

When $p 66 \beta$ knockdown cells were treated with a high dose of $\mathrm{Hh}$, induced $\mathrm{Hh}$ activity was similar to that in wild-type cells (Supplemental Fig. S13). We reason that this was because Sufu dissociated from Gli (this occurs largely in the nucleus in our model) using high doses of Hh ligand. Under this condition, the level of p $66 \beta$, whose function in Hh signaling depends on Sufu, would make no difference on $\mathrm{Hh}$ responses. Hence, $p 66 \beta$ knockdown exerts little effect on Gli if Sufu/Gli dissociation has already occurred. In contrast, when cells are subjected to submaximal Hh stimulation, Sufu is not expected to completely dissociate from Gli in the nucleus. As expected, increased Hh responses were observed in $p 66 \beta$ knockdown cells.

We showed that HDAC1 was coimmunoprecipitated with $\mathrm{p} 66 \beta$ and Sufu, but Sufu did not pull down other known components of the $\mathrm{NuRD}$ repressor. It is possible that interactions between p66//Sufu and other NuRD components are weak or transient. However, it is also possible that the $\mathrm{p} 66 \beta / \mathrm{Sufu}$ interaction represents a novel function of $\mathrm{p} 66 \beta$ independent of the NuRD complex. Interestingly, HDACl also exhibits NuRD-independent function (Canettieri et al. 2010). Whether p66 $\beta$ recruits additional components or relies on post-translational modifications of $\mathrm{p} 66 \mathrm{\beta}$ and Gli proteins to control $\mathrm{Hh}$ signaling is unknown and would rely on future biochemical studies.

The phenotypes associated with the loss of $p 66 \beta$ in mice are currently unavailable and would require the production of mutant mice and phenotypic analysis. Nevertheless, it is interesting to note that knockout mice of $p 66 \alpha$ die at $9.5 \mathrm{~d}$ post-coitus (dpc) (Marino and Nusse 2007). The observation that the loss of $p 66 \alpha$ does not lead to global cell death at the earliest stage of embryonic development implies that components of the NuRD complex could be involved in specific cellular processes or interact with particular pathways during development. It is also plausible that functional redundancy exists between $p 66 \beta$ and other components, and a better mechanistic understanding of $p 66 \beta$ function is required for proper interpretation of the phenotypes.

\section{Regulation of Gli activity by Sufu and Mycbp}

We showed that Mycbp enhances Hh signaling in cultured cells and zebrafish. Mycbp was initially identified as a Mycinteracting protein and is known to enhance the activity of Myc (Taira et al. 1998). Mycbp displays cell cycle-dependent shuttling between the nucleus and the cytoplasm (Furusawa et al. 2002). In addition, overexpressed Mycbp can be detected in both the nucleus and cytoplasm. While Mycbp is present on the promoter of Hh-responsive genes, we cannot rule out its potential roles in cytoplasmic $\mathrm{Hh}$ signaling. In this regard, it is interesting to note that Mycbp could also be detected at the base of the primary cilium 
(Fig. 2H). Mycbp has been previously shown to bind to modulators of PKA and suppress PKA activity (Furusawa et al. 2002). PKA negatively regulates $\mathrm{Hh}$ signaling in a cilium-dependent manner (Tuson et al. 2011). Whether Mycbp exerts its activity on Hh signaling at the cilium base is unknown, although overexpression of Mycbp fails to perturb ciliary distributions of Gli proteins.

Studies in cultured cells and zebrafish support Mycbp's role as a novel positive regulator of Hh signaling. Similarly, insight into the in vivo function of Mycbp in mammalian Hh signaling would need to await loss-of-function studies in mice. In this case, conditional inactivation of Mycbp may be necessary to overcome its early lethality caused by its pleiotropic effects unrelated to Hh signaling. Alternatively, double-mutant analysis may be required if functional redundancy exists between Mycbp and other components. Finally, mapping the functional domains of $\mathrm{p} 66 \beta$ and Mycbp could facilitate the production of mutants that have selective disruption of Hh signaling.

\section{p66 $\beta$ and Mycbp on the chromatin of Hh target genes}

Our ChIP analysis revealed the presence of $\mathrm{p} 66 \beta$ and Mycbp at the promoter region of $\mathrm{Hh}$ target genes. In addition, p66 $\beta$ interactions with Gli depend on Sufu, while Sufu's presence on $\mathrm{Hh}$ promoters requires Gli proteins. These results support our model in which Sufu recruits p $66 \beta$ to inhibit Gli activity without Hh signaling. We propose that Hh stimulation leads to dissociation of the Sufu/p66ß complex from Gli proteins and relieves Gli inhibition, since Sufu/p $66 \beta$ dissociates from Gli proteins, while the Sufu/p66 interaction does not seem to be affected by $\mathrm{Hh}$ signaling. Consistent with this model, $\mathrm{Hh}$ pathway activation results in a concomitant reduction in occupancy on Hh-responsive promoters for both Sufu and p66 3 . In addition, we demonstrated that Sufu protein levels in the nucleus decrease upon $\mathrm{Hh}$ activation, and this could contribute to the relief of Gli inhibition by Sufu. Regulation of Sufu activity at multiple levels ensures a tight control of Gli protein function.

Our coimmunoprecipitation studies suggest that Mycbp interacts with Sufu and Gli in the absence of Hh signaling. This led to our model that Gli (and/or Sufu) recruits Mycbp to Hh targets, but Mycbp remains inactive. When $\mathrm{Hh}$ stimulation promotes dissociation of the Sufu/p66 $\mathrm{B}$ complex from Gli proteins, Mycbp's association with Hh promoters is enhanced, which is correlated with increased binding between Mycbp and Gli. This would confer the ability of Mycbp to stimulate Gli activity. How Mycbp is inactivated without Hh signaling and how Mycbp activity is switched on upon Hh pathway activation remain unclear. We suspect that this could involve the recruitment of other proteins or post-translational modifications of Mycbp and Gli proteins as a result of $\mathrm{Hh}$ signaling. Biochemical studies are required to address these critical issues.

We did not have the spatial or temporal resolution to detect real-time changes of $\mathrm{p} 66 \beta$ and Mycbp on $\mathrm{Hh}$ promoters. Consequently, we have no insight into the dynamic behaviors of $p 66 \beta$ and Mycbp on the chromatin during $\mathrm{Hh}$ target gene activation. For instance, while we show that Sufu/p66 3 are released from the Hh promoters upon pathway activation, Sufu/p66 $\beta$ may subsequently be recruited to Hh promoters during active transcription. This could provide a means to efficiently turn off $\mathrm{Hh}$ signaling. Gaining insight into these mechanistic issues would require using new technologies to study the dynamics of these proteins on Hh promoters during Hh pathway activation. It is also important to note that the dynamic interactions among p $66 \beta$, Mycbp, and Gli proteins in response to Hh signaling follow a quantitative and not an all-or-none change. This may endow the system with the ability to produce graded $\mathrm{Hh}$ responses.

It is possible that Sufu/p66ß/Mycbp controls only a subset of Hh targets or that multiple mechanisms are used to control Hh target gene expression. This would suggest that additional regulators other than $\mathrm{p} 66 \beta / \mathrm{Mycbp}$ may be needed to control $\mathrm{Hh}$ target gene expression, and functional redundancy may exist to ensure tight control of $\mathrm{Hh}$ target gene expression. In fact, Sufu has been reported to associate with the SAP18-mSin3 repressor complex/Cheng and Bishop 2002), although its relevance has not been validated in vitro (Chen et al. 2009) or in vivo.

\section{p66 $\beta$ and Mycbp in Hh signaling in diverse species}

Our analysis of Sufu, p66 $\beta$, and Mycbp in the mammalian system suggests that these new Sufu regulators function in vertebrate Hh signaling. Whether they also play a role in invertebrate Hh signaling is not known. Interestingly, the Drosophila homolog of $p 66$ was identified as a modifier of $\mathrm{Wg}$ signaling. Loss of $p 66$ in fly wing and eye discs does not have detectable phenotypes, a result attributed to its redundancy with other histone deacetylase complexes (Kon et al. 2005). The biochemical function of Sufu appears to be conserved in $\mathrm{Hh}$ signaling in different species. Sufu sequesters Ci/Gli proteins and controls their protein levels and repressor formation, and studies in Drosophila also support a role of $\mathrm{Su}(\mathrm{fu})$ in suppressing nuclear activity of $\mathrm{Ci}$ independent of its effects on nuclear import of Ci (Wang et al. 2000b). Nevertheless, Sufu could be subjected to distinct modes of regulation in each organism. It is thus possible that $\mathrm{p} 66 \beta$ and Mycbp represent vertebrate-specific regulators of Sufu and reflect pathway divergence.

\section{p66 $\beta$ and Mycbp as potential targets of manipulating Hh pathway activity}

The identification of $\mathrm{p} 66 \beta$ and Mycbp as regulators of Sufu and Gli activity in vertebrate $\mathrm{Hh}$ signaling also provides new targets of modulating $\mathrm{Hh}$ activity. In particular, if the molecular interfaces between p $66 \beta$ and Mycbp and Sufu/Gli could be identified, this would allow screening of molecules that can manipulate Hh signaling without affecting other processes in which p $66 \beta$ and Mycbp may participate. Many disease processes may result from unregulated Gli activity that could even be independent of upstream $\mathrm{Hh}$ components. In this regard, new modulators of Gli protein function, such as $\mathrm{p} 66 \beta$ and Mycbp, offer a unique opportunity to develop new therapies for diseases due to aberrant Gli activity. 


\section{Materials and methods}

\section{Cell lines and constructs}

MEFs stably expressing 3xFlag-Sufu, GFP-Sufu ${ }^{\mathrm{N} 100}$, GFP-Sufu ${ }^{\mathrm{N} 212}$, $3 x F l a g-p 66 \beta$, 3xFlag-Mycbp, 3xFlag-Gli2, and 3x-Flag-Gli3 were generated through retroviral transduction as described (Chen et al. 2009). Clones with stable expression of Sufu were confirmed by Western blotting. HEK293T and NIH3T3 cells and transformed MEF lines were maintained in DMEM supplemented with $10 \%$ fetal bovine serum (FBS), penicillin/streptomycin, and L-glutamine (Life Technologies). Wild-type, Sufu ${ }^{-/}, \mathrm{Ptch}^{-/-}, \mathrm{Gli2}^{-/-}, \mathrm{Gli3}^{-/-}$, and $K i f 3 a^{-1-}$ MEFs have been previously described (Chen et al. 2009) and were maintained in medium containing $500 \mu \mathrm{g} / \mathrm{mL}$ G418. Gli2 ${ }^{-1-}$; Gli3 ${ }^{-1-}$ MEFs (Zeng et al. 2010) were kindly provided by Aimin Liu. Kif $7^{-1-}$ MEFs were derived from Kif7deficient embryos (Cheung et al. 2009) as previously reported (Chen et al. 2009).

Standard molecular biology was used to construct mouse FlagSufu $^{\mathrm{N} 100}$, Flag-Sufu ${ }^{\mathrm{N} 212}$, Flag-p66 $\beta$, Myc-p66 $\beta$, Flag-Mycbp, and Myc-Mycbp. Flag-Sufu, Myc-Sufu, Flag-Gli1, Flag-Gli2, and FlagGli3 have been reported (Chen et al. 2009).

Full-length mouse and zebrafish p $66 \beta$ and Mycbp cDNAs were C-terminally tagged with $3 x$ Flag, 6xMyc, or GFP and cloned into pcDNA3 or pCS2 ${ }^{+}$for transient overexpression in cultured cells or zebrafish embryos and into pBABE-puro for retroviral overexpression in cultured cells. Mouse and zebrafish p66 $\beta$ and Mycbp cDNAs were also C-terminally tagged with GFP and cloned into $\mathrm{pCS}^{+}$for transient expression in zebrafish embryos.

\section{Affinity purification and mass spectrometry}

Ten 10-cm plates of MEFs stably expressing Sufu-3xFlag were cultured to confluence. Cells were starved in 0.5\% FBS/DMEM for $12 \mathrm{~h}$ and then switched to $0.5 \%$ FBS/DMEM with mock- or Shh-conditioned medium for $24 \mathrm{~h}$. Cells were lysed in $1.5 \mathrm{~mL}$ of cold lysis buffer $(50 \mathrm{mM}$ Tris- $\mathrm{Cl}$ at $\mathrm{pH} 7.5,150 \mathrm{mM} \mathrm{NaCl}, 1 \mathrm{mM}$ EDTA, $0.5 \%$ Nonidet P40 substitute, complete protease inhibitor [Roche], PhosSTOP [Roche]). Cells were dounced 20 times on ice and spun at $3400 \mathrm{rpm}$ for $20 \mathrm{~min}$. The supernatant was incubated with $60 \mu \mathrm{L}$ of preclearing beads (mouse IgG agarose; Sigma) for $2 \mathrm{~h}$, and the precleared lysate was incubated with $30 \mu \mathrm{L}$ of Flag M2 beads (Sigma) overnight. The beads were washed four times with lysis buffer containing $0.2 \%$ Nonidet P40 substitute followed by three times with lysis buffer without detergents. Proteins were eluted with $40 \mu \mathrm{L}$ of $50 \mathrm{mM}$ Tris-Cl (pH 7.5), $150 \mathrm{mM} \mathrm{NaCl}, 1 \mathrm{mM}$ EDTA containing $100 \mu \mathrm{g} / \mathrm{mL}$ $3 x$ Flag peptide (ELIM), and $0.05 \%$ RapiGest (Waters). One microliter of the eluate was analyzed by Western blotting with anti-Flag (1:2000; Sigma), anti-Gli2 (1:500; R\&D Systems), and anti-Gli3 (R\&D, 1:500) antibodies. Ten microliters of eluate was analyzed by Coomassie Blue and $2 \mu \mathrm{L}$ was analyzed by silver staining.

Mass spectrometry was performed essentially as described (Jager et al. 2011). Briefly, for gel-free mass spectrometry, $10 \mu \mathrm{L}$ of the eluate from immunoprecipitation was reduced, carboxyamidomethylated, and digested with trypsin. For gel-based analysis, $30 \mu \mathrm{L}$ of eluate was separated by $7 \%$ SDS-PAGE and stained with Coomassie Blue. The band of interest was cut out. Each gel piece was diced into small pieces, which were reduced, carboxyamidomethylated, and digested with trypsin. All samples were analyzed on a Thermo Scientific LTQ Orbitrap XL mass spectrometer equipped with a nanoACQUITY UPLC (Waters) chromatography system and a nanoelectrospray source. The data-dependent mass spectrometer continuously collected a survey scan in the Orbitrap mass analyzer at 40,000 resolution with an automatic gain control (AGC) target of $1 \times 10^{6}$ followed by collision-induced dissociation
(CID) tandem mass spectrometry scans of the 10 most abundant ions in the survey scan in the ion trap with an AGC target of 5000, a signal threshold of 1000, a 2.0-Da isolation width, and a 30-msec activation time at $35 \%$ normalized collision energy. Raw mass spectrometric data were converted into peak lists using Bioworks 3.3.1 SP1. The spectra were searched using Prospector version 5.3 (http://prospector.ucsf.edu) against a mouse-restricted UniProt database. Protein Prospector results were filtered by applying a minimum protein score of 22.0, a minimum peptide score of 15.0, a maximum protein $E$-value of 0.01 , and a maximum peptide $E$-value of 0.05 .

\section{Nuclear-cytoplasmic fractionation}

Subcellular fractionation was performed as reported (Chen et al. 2009). The purity of the cytoplasmic and nuclear fractions was assessed by cytoplasmic- and nuclear-specific markers, including anti-tubulin (1:5000; Sigma) and anti-Lamin A (1:3000; Abcam). The following antibodies were used for Western blotting of cytoplasmic and nuclear fractions: rabbit anti-Sufu (1:3000; Santa Cruz Biotechnology), goat anti-Gli2 (1:500; R\&D Systems), and goat anti-Gli3 (1:500; R\&D Systems).

\section{Coimmunoprecipitation}

Different combinations of Myc-Mycbp, Myc-p66 $\beta$, Flag-Sufu, FlagGli1, and Flag-Gli2 were transfected into HEK293T cells by Lipofectamine 2000 (Life Technologies). Cells were collected at $48 \mathrm{~h}$ post-transfection and lysed in immunoprecipitation buffer (1\% Triton X-100, $150 \mathrm{mM} \mathrm{NaCl}, 50 \mathrm{mM}$ Tris-Cl at pH 7.5, $1 \mathrm{mM}$ EDTA, protease inhibitor cocktail [Roche], PhosSTOP [Roche]). The lysates were cleared by centrifugation at $12,000 \mathrm{rpm}$ for $10 \mathrm{~min}$ at $4^{\circ} \mathrm{C}$. The supernatant was removed and bound to $20 \mu \mathrm{L}$ of antiFlag M2 beads (Sigma) or anti-Myc beads (Santa Cruz Biotechnology) overnight at $4^{\circ} \mathrm{C}$ with constant nutation. Beads were washed three times with immunoprecipitation buffer and eluted with SDS sample buffer. Immunoprecipitates were analyzed by Western blotting using rabbit anti-Flag (1:2000; Sigma) and rabbit anti-Myc (1:2000; Sigma).

For immunoprecipitation of endogenous Sufu, Gli2, and Gli3, a 10-cm plate of confluent wild-type MEFs or NIH3T3 fibroblasts were starved in $0.5 \%$ FBS/DMEM for $12 \mathrm{~h}$ and then treated with Shh-conditioned medium or $100 \mathrm{mM} \mathrm{SAG}$ (Sigma) in $0.5 \%$ FBS/DMEM for the indicated time. Cells were lysed in immunoprecipitation buffer. The lysate was precleared by incubating with protein A or protein G beads, and primary antibody was subsequently added and incubated overnight at $4^{\circ} \mathrm{C}$. Protein A (for anti-Sufu antibody) or protein $\mathrm{G}$ (for anti-Gli antibody) beads were added to the lysate. After incubation overnight at $4^{\circ} \mathrm{C}$, the beads were washed with immunoprecipitation buffer and eluted with SDS sample buffer. The antibodies used were rabbit antiSufu (1:3000; Santa Cruz Biotechnology), rabbit anti-Gli2 (1:500; R\&D Systems), and goat anti-Gli3 (1:500; R\&D Systems).

For immunoprecipitation of wild-type MEFs stably expressing Flag-tagged Sufu, Gli1, Gli2, or Gli3 and Sufu ${ }^{-1}$ MEFs stably expressing Flag-tagged Gli2, Gli3, or Mycbp, cells were lysed in immunoprecipitation buffer. The lysate was centrifuged and then precleared by incubating with $30 \mu \mathrm{L}$ of mouse IgG agarose beads (Sigma) in a $50 \%$ slurry for $1 \mathrm{~h}$ at $4^{\circ} \mathrm{C}$. The lysate was subsequently incubated with $20 \mu \mathrm{L}$ of Flag M2 agarose beads (Sigma) in a $50 \%$ slurry overnight at $4{ }^{\circ} \mathrm{C}$. The beads were washed rigorously in immunoprecipitation buffer and eluted with SDS sample buffer. Immunoprecipitates were analyzed by Western blotting using the following antibodies: rabbit anti-p66 $\beta$ (1:500; Millipore), rabbit anti-HDAC1 (1:500; Santa Cruz Biotechnology), rabbit anti-HDAC2 (1:1000; Santa Cruz Biotechnology), goat anti-Rbap46/48 (1:500; Santa Cruz Biotechnology), rabbit anti- 
Lin et al.

MBD2/3 (1:200; Santa Cruz Biotechnology), and goat anti-MTA1 (1:500; Santa Cruz Biotechnology).

\section{shRNA-mediated knockdown}

shRNAs were designed using pSicOligomaker (Reynolds et al. 2004), and oligonucleotides encoding shRNAs were cloned into the pLentiLox3.7 vector. Lentiviruses were produced as described (Chen et al. 2009). MEFs at 50\% confluence were transduced with lentiviruses supplemented with $8 \mathrm{mg} / \mathrm{mL}$ polybrene. Upon reaching confluence, cells were starved in 0.5\% FBS/DMEM for $12 \mathrm{~h}$ and switched to $0.5 \% \mathrm{FBS} / \mathrm{DMEM} / \mathrm{Sh}$-conditioned medium for another $24 \mathrm{~h}$. The following 19-mer sequences were used for shRNA-mediated knockdown: mouse Mycbp (NM_019660), 5' GCGTTCTGGTTGCACATTA-3' and 5'-GCAGCTACCTTGA TTGTAA-3'; mouse $p 66 \beta$ (NM_139304), 5'-GCAGTGGTGTC AAGGGTTA-3' and 5'-GCAGCAGCTTATCAAGCAA-3'; mouse Septin 11 (NM_001009818), 5'-GCAACGGAGTCCAGATATA-3'; mouse Prmt5 (NM_013768), 5' -GGCAGATGGATCTCTTTAA-3'; and GFP, 5'-GCAGACCATTATCAACAAA-3'. shRNA against GFP was used as a control.

For Mycbp knockdown, both sets of shRNA were used in combination. For $p 66 \beta$ knockdown, shRNA directed against the 5'-GCAGCAGCTTATCAAGCAA-3' target sequences was used in most of the data presented. An shRNA-resistant $p 66 \beta$ cDNA $\left(p 66 \beta^{r}\right)$ was generated by changing the $5^{\prime}$-GCAGCAGCTTATC AAGCAA-3' target sequences to 5' -ACAACAACTCATTAAGC AA-3' without altering the amino acids encoded.

The following 19-mer sequences were used for shRNAmediated knockdown of mouse NuRD complex components: p66 $\alpha$ (NM_145596), 5' -GTACAGTTAGGGACTTTAA-3' ; HDAC1 (NM_008228), 5'-GAAGAGGCCTTCTATACTA-3' and 5'-GAAC TCTTCTAACTTCAAA-3'; HDAC2 (NM_008229), 5'-GAATCC GGATGACTCATAA-3'; MBD2 (NM_010773), 5'-GTAGCACTT ACGTGAAATA-3'; and Rbbp4 (NM_009030), 5' -GAAATTGGCT GCCCTTTGA-3'.

\section{Immunofluorescence}

Cells were fixed in $4 \%$ paraformaldehyde for $15 \mathrm{~min}$ at room temperature. Standard procedures for immunostaining were followed. The primary antibodies used were mouse anti-acetylated tubulin (1:2000; Sigma), rabbit anti-Flag (1:2000; Sigma), and rabbit anti-Myc (1:1000; Sigma). Secondary antibodies and conjugates used were donkey anti-mouse Alexa Fluor 594 (1:2000; Life Technologies), donkey anti-rabbit Alexa Fluor 488 (1:2000; Life Technologies), and DAPI (1:10,000; Sigma).

\section{qPCR analysis}

RNA was extracted from NIH3T3 cells or MEFs using Trizol (Life Technologies) and reverse-transcribed to the first strand cDNA using Maxima Reverse Transcriptase (Thermo Scientific). Real-time qPCR was carried out in an ABI Prism 7900HT system using FastStart SYBR Green master mix (Roche). Relative gene expression data were analyzed using the $2^{-\Delta \Delta \mathrm{CT}}$ method (Schmittgen and Livak 2008). GAPDH was used for normalization of gene expression. The primers used were mouse Ptch1 (forward, 5' -TGCTGTGCCTGTGGTCATCCTGATT-3'; reverse, 5'-CAGAGCGAGCATAGCCCTGTGGTTC-3') and mouse Gli1 (forward, 5' -CCCATAGGGTCTCGGGGTCTCAAAC-3'; reverse, 5'-GGAGGACCTGCGGCTGACTGTGTAA-3').

\section{Hh and Wnt reporter assays}

For measuring Hh pathway activities, NIH3T3 cells or MEFs were transfected with a 4:5:1 ratio of pcDNA3.1/expression
construct:8xGliBS-luc:pRL-TK using Lipofectamine 2000 (Life Technologies). For assessing Wnt pathway activation, NIH3T3 cells or MEFs were transfected with a 4:5:1 ratio of pcDNA3.1/ Wnt3a expression construct:TOPFlash:pRL-TK. Luciferase assays were performed as described (Chen et al. 2009).

\section{ChIP}

ChIP was performed using the EZ-ChIP kit (Upstate Biotechnology) according to the manufacturer's manual. Briefly, cells were cross-linked in $1 \%$ formaldehyde, and the DNA was sonicated into a range of 100-600 base pairs (bp) in size using a Bioruptor Sonicator (Diagenode) for five cycles of $30 \mathrm{sec}$ on/30 sec off. The extracts were precleared in BSA-blocked protein G and incubated with antibodies or IgG control overnight. After washes, the DNA was eluted and reverse-cross-linked overnight at $65^{\circ} \mathrm{C}$. The DNA was purified and analyzed by qPCR. The antibodies used were mouse anti-Flag M2 monoclonal antibody (Sigma) and normal mouse IgG (Santa Cruz Biotechnology). The primers for qPCR were mouse Ptch1 promoter (forward, 5'-TTATAAAGCA GGTCCCCAAC-3'; reverse, 5'-ACGCATGTTTGCAAAGTA GA-3'), mouse Gli1 promoter (forward, 5'-AGGAGATGCTCTG ACGCCTA-3'; reverse, 5'-GGACAAAGAGACCTGGGACA-3'), mouse Hhip (Hip1) promoter (forward, 5' -AATTGCTGCAGACCC TAAAT-3'; reverse, $5^{\prime}$-TAAAAGGGCACACTTGAAAA- ${ }^{\prime}$ ), and mouse $\beta$-actin promoter (forward, 5'-AGAAGGACTCCTATGT GGGTGA-3'; reverse, 5' -ACTGACCTGGGTCATCTTTTC-3').

\section{Zebrafish mRNA injection}

For mRNA synthesis, plasmids were linearized by NotI, and mRNA was synthesized using the SP6 mMESSAGE mMachine kit according to the manufacturer's manual (Ambion). Threehundred picograms of $p 66 \beta-6 x M y c, p 66 \beta-G F P$, mycbp-6xMyc, and mycbp-GFP mRNA (in pCS2 $2^{+}$vector) was injected into onecell zebrafish embryos (Langenbacher et al. 2012). Embryos were collected at $24 \mathrm{~h}$ post-fertilization (hpf), $36 \mathrm{hpf}$, and $3.5 \mathrm{~d}$ postfertilization. To avoid variations in expression levels, in some experiments, we also selected injected embryos expressing strong GFP at 8 and 20 hpf for subsequent analysis. Embryos were fixed at $32 \mathrm{hfp}$ for whole-mount in situ hybridization using patched1 (ptch1) riboprobe and standard procedures. ptch1 riboprobe was synthesized using ptch1 cDNA cloned into pCS2 ${ }^{+}$. Mouse and zebrafish p $66 \beta$ and Mycbp yielded similar results, although zebrafish homologs produced more robust phenotypes. p66 $\beta$ or mycbp tagged with GFP at the $3^{\prime}$ end produced results similar to those of untagged $\mathrm{p} 66 \beta$ or mycbp. Results in Figure 4 were derived from embryos injected with zebrafish $p 66 \beta$-GFP and mycbp-GFP mRNA.

\section{MO-mediated knockdown in zebrafish}

Zebrafish $p 66 \beta$ antisense oligos (5'-CCTCCTCAGACATCCGC TCCATCCT-3') and mycbp antisense oligos (5'-GCGATGGCG CATTACAGA-3') were synthesized by GeneTools, LLC. One nanogram of $p 66 \beta \mathrm{MO}$ was injected into one-cell stage zebrafish embryos, which were fixed at $36 \mathrm{hpf}$ for in situ hybridization analysis. Similarly, $4 \mathrm{ng}$ of mycbp MO was injected into one-cell stage embryos harvested at $36 \mathrm{hpf}$ for in situ hybridization.

\section{Acknowledgments}

We thank Cecilia Huang for technical assistance, Aimin Liu for cell lines, C.C. Hui for Kif7 mutant mice, the University of California at Los Angeles Statistical Consulting Group for advice on z-tests, and Thermo Scientific for providing instruments for 
mass spectrometry. Some data for this study were acquired at the Nikon Imaging Center at the Cardiovascular Research Institute (CVRI). This work was supported by grants from the National Institutes of Health to J.-N.C. (R01 HL081700 and R01 HL096980), N.J.K. (P50 GM081879 and R01 GM084279) and P.-T.C. (R01 GM085097).

\section{References}

Aberger F, Ruiz i Altaba A. 2014. Context-dependent signal integration by the GLI code: the oncogenic load, pathways, modifiers and implications for cancer therapy. Semin Cell Dev Biol 33: 93-104.

Allen HF, Wade PA, Kutateladze TG. 2013. The NuRD architecture. Cell Mol Life Sci 70: 3513-3524.

Altelaar AF, Munoz J, Heck AJ. 2013. Next-generation proteomics: towards an integrative view of proteome dynamics. Nat Rev Genet 14: 35-48.

Bai CB, Stephen D, Joyner AL. 2004. All mouse ventral spinal cord patterning by hedgehog is Gli dependent and involves an activator function of Gli3. Dev Cell 6: 103-115.

Barakat MT, Humke EW, Scott MP. 2010. Learning from Jekyll to control Hyde: Hedgehog signaling in development and cancer. Trends Mol Med 16: 337-348.

Barnfield PC, Zhang X, Thanabalasingham V, Yoshida M, Hui CC. 2005. Negative regulation of Gli1 and Gli2 activator function by Suppressor of fused through multiple mechanisms. Differentiation 73: 397-405.

Bay SN, Caspary T. 2012. What are those cilia doing in the neural tube? Cilia 1: 19.

Beachy PA, Hymowitz SG, Lazarus RA, Leahy DJ, Siebold C. 2010. Interactions between Hedgehog proteins and their binding partners come into view. Genes Dev 24: 20012012.

Berbari NF, O'Connor AK, Haycraft CJ, Yoder BK. 2009. The primary cilium as a complex signaling center. Current Biol 19: R526-R535.

Bijlsma MF, Roelink H. 2010. Non-cell-autonomous signaling by Shh in tumors: challenges and opportunities for therapeutic targets. Expert Opin Ther Targets 14: 693-702.

Bowers M, Eng L, Lao Z, Turnbull RK, Bao X, Riedel E, Mackem S, Joyner AL. 2012. Limb anterior-posterior polarity integrates activator and repressor functions of GLI2 as well as GLI3. Dev Biol 370: 110-124.

Briscoe J, Therond PP. 2013. The mechanisms of Hedgehog signalling and its roles in development and disease. Nat ReV Mol Cell Biol 14: 416-429.

Canettieri G, Di Marcotullio L, Greco A, Coni S, Antonucci L, Infante P, Pietrosanti L, De Smaele E, Ferretti E, Miele E, et al. 2010. Histone deacetylase and Cullin3-REN(KCTD11) ubiquitin ligase interplay regulates Hedgehog signalling through Gli acetylation. Nat Cell Biol 12: 132-142.

Cao T, Wang C, Yang M, Wu C, Wang B. 2013. Mouse limbs expressing only the Gli3 repressor resemble those of Sonic hedgehog mutants. Dev Biol 379: 221-228.

Chen Y, Jiang J. 2013. Decoding the phosphorylation code in Hedgehog signal transduction. Cell Res 23: 186-200.

Chen MH, Wilson CW, Li YJ, Law KK, Lu CS, Gacayan R, Zhang X, Hui CC, Chuang PT. 2009. Cilium-independent regulation of Gli protein function by Sufu in Hedgehog signaling is evolutionarily conserved. Genes Dev 23: 19101928.

Chen Y, Yue S, Xie L, Pu XH, Jin T, Cheng SY. 2011. Dual phosphorylation of suppressor of fused (Sufu) by PKA and GSK3 $\beta$ regulates its stability and localization in the primary cilium. I Biol Chem 286: 13502-13511.
Cheng SY, Bishop JM. 2002. Suppressor of Fused represses Glimediated transcription by recruiting the SAP18-mSin 3 corepressor complex. Proc Natl Acad Sci 99: 5442-5447.

Cherry AL, Finta C, Karlstrom M, Jin Q, Schwend T, AstorgaWells J, Zubarev RA, Del Campo M, Criswell AR, de Sanctis D, et al. 2013. Structural basis of SUFU-GLI interaction in human Hedgehog signalling regulation. Acta Crystallogr D Biol Crystallogr 69: 2563-2579.

Cheung HO, Zhang X, Ribeiro A, Mo R, Makino S, Puviindran V, Law KK, Briscoe J, Hui CC. 2009. The kinesin protein Kif7 is a critical regulator of Gli transcription factors in mammalian hedgehog signaling. Sci Signal 2: ra29.

Chuang PT, McMahon AP. 1999. Vertebrate Hedgehog signalling modulated by induction of a Hedgehog-binding protein. Nature 397: 617-621.

Collas P. 2011. A chromatin immunoprecipitation protocol for small cell numbers. Methods Mol Biol 791: 179-193.

Cooper AF, Yu KP, Brueckner M, Brailey LL, Johnson L, McGrath JM, Bale AE. 2005. Cardiac and CNS defects in a mouse with targeted disruption of suppressor of fused. Development 132: 4407-4417.

Corbit KC, Aanstad P, Singla V, Norman AR, Stainier DY, Reiter JF. 2005. Vertebrate Smoothened functions at the primary cilium. Nature 437: 1018-1021.

DeRouen MC, Oro AE. 2009. The primary cilium: a small yet mighty organelle. J Invest Dermatol 129: 264-265.

Di Marcotullio L, Greco A, Mazza D, Canettieri G, Pietrosanti L, Infante P, Coni S, Moretti M, De Smaele E, Ferretti E, et al. 2011. Numb activates the E3 ligase Itch to control Glil function through a novel degradation signal. Oncogene 30: 65-76.

Ding Q, Motoyama J, Gasca S, Mo R, Sasaki H, Rossant J, Hui CC. 1998. Diminished Sonic hedgehog signaling and lack of floor plate differentiation in Gli2 mutant mice. Development 125: 2533-2543.

Ding Q, Fukami S, Meng X, Nishizaki Y, Zhang X, Sasaki H, Dlugosz A, Nakafuku M, Hui C 1999. Mouse suppressor of fused is a negative regulator of sonic hedgehog signaling and alters the subcellular distribution of Gli1. Curr Biol 9: 11191122.

Drummond IA. 2012. Cilia functions in development. Curr Opin Cell Biol 24: 24-30.

Eggenschwiler JT, Anderson KV. 2007. Cilia and developmental signaling. Annu Rev Cell Dev Biol 23: 345-373.

Endoh-Yamagami S, Evangelista M, Wilson D, Wen X, Theunissen JW, Phamluong K, Davis M, Scales SJ, Solloway MJ, de Sauvage FJ et al. 2009. The mammalian Cos2 homolog Kif7 plays an essential role in modulating $\mathrm{Hh}$ signal transduction during development. Curr Biol 19: 1320-1326.

Evangelista M, Lim TY, Lee I, Parker L, Ashique A, Peterson AS, Ye W, Davis DP, de Sauvage FJ. 2008. Kinome siRNA screen identifies regulators of ciliogenesis and hedgehog signal transduction. Sci Signal 1: ra7.

Falkenstein KN, Vokes SA. 2014. Transcriptional regulation of graded Hedgehog signaling. Semin Cell Dev Biol 33: 73-80.

Farzan SF, Singh S, Schilling NS, Robbins DJ. 2008. The adventures of sonic hedgehog in development and repair. III. Hedgehog processing and biological activity. Am J Physiol Gastrointest Liver Physiol 294: G844-G849.

Filmus J, Capurro M 2014. The role of glypicans in Hedgehog signaling. Matrix Biol 35C: 248-252.

Furusawa M, Taira T, Iguchi-Ariga SM, Ariga H. 2002. AMY-1 interacts with S-AKAP84 and AKAP95 in the cytoplasm and the nucleus, respectively, and inhibits cAMP-dependent protein kinase activity by preventing binding of its catalytic subunit to A-kinase-anchoring protein (AKAP) complex. J Biol Chem 277: 50885-50892. 
Goetz SC, Anderson KV. 2010. The primary cilium: a signalling centre during vertebrate development. Nat Rev Genet 11: 331-344.

Goodrich LV, Johnson RL, Milenkovic L, McMahon JA, Scott MP. 1996. Conservation of the hedgehog/patched signaling pathway from flies to mice: induction of a mouse patched gene by Hedgehog. Genes Dev 10: 301-312.

Hannon GJ. 2003. RNAi: a guide to gene silencing. Cold Spring Harbor Laboratory Press, Cold Spring Harbor, NY.

Harrison MM, Jenkins BV, O'Connor-Giles KM, Wildonger J. 2014. A CRISPR view of development. Genes Dev 28: 1859-1872.

Haycraft CJ, Banizs B, Aydin-Son Y, Zhang Q, Michaud EJ, Yoder BK. 2005. Gli2 and Gli3 localize to cilia and require the intraflagellar transport protein polaris for processing and function. PLoS Genet 1: e53.

Hillman RT, Feng BY, Ni J, Woo WM, Milenkovic L, Hayden Gephart MG, Teruel MN, Oro AE, Chen JK, Scott MP. 2011. Neuropilins are positive regulators of Hedgehog signal transduction. Genes Dev 25: 2333-2346.

Hui CC, Angers S. 2011. Gli proteins in development and disease. Annu Rev Cell Dev Biol 27: 513-537.

Humke EW, Dorn KV, Milenkovic L, Scott MP, Rohatgi R. 2010. The output of Hedgehog signaling is controlled by the dynamic association between Suppressor of Fused and the Gli proteins. Genes Dev 24: 670-682.

Ingham PW, Nakano Y, Seger C. 2011. Mechanisms and functions of Hedgehog signalling across the metazoa. Nat Rev Genet 12: 393-406.

Jacob LS, Wu X, Dodge ME, Fan CW, Kulak O, Chen B, Tang W, Wang B, Amatruda JF, Lum L. 2011. Genome-wide RNAi screen reveals disease-associated genes that are common to Hedgehog and Wnt signaling. Sci Signal 4: ra4.

Jager S, Gulbahce N, Cimermancic P, Kane J, He N, Chou S, D'Orso I, Fernandes J, Jang G, Frankel AD, et al. 2011. Purification and characterization of HIV-human protein complexes. Methods 53: 13-19.

Jia J, Kolterud A, Zeng H, Hoover A, Teglund S, Toftgard R, Liu A. 2009. Suppressor of Fused inhibits mammalian Hedgehog signaling in the absence of cilia. Dev Biol 330: 452-460.

Kim S, Dynlacht BD. 2013. Assembling a primary cilium. Curr Opin Cell Biol 25: 506-511.

Kim J, Kato M, Beachy PA. 2009. Gli2 trafficking links Hedgehogdependent activation of Smoothened in the primary cilium to transcriptional activation in the nucleus. Proc Natl Acad Sci 106: $21666-21671$.

Kogerman P, Grimm T, Kogerman L, Krause D, Unden AB, Sandstedt B, Toftgard R, Zaphiropoulos PG. 1999. Mammalian suppressor-of-fused modulates nuclear-cytoplasmic shuttling of Gli-1. Nat Cell Biol 1: 312-319.

Kon C, Cadigan KM, da Silva SL, Nusse R. 2005. Developmental roles of the Mi-2/NURD-associated protein p66 in Drosophila. Genetics 169: 2087-2100.

Kornberg TB. 2014. The contrasting roles of primary cilia and cytonemes in Hh signaling. Dev Biol 394: 1-5.

Kuzhandaivel A, Schultz SW, Alkhori L, Alenius M. 2014. Ciliamediated hedgehog signaling in Drosophila. Cell Reports 7: 672-680.

Lai AY, Wade PA. 2011. Cancer biology and NuRD: a multifaceted chromatin remodelling complex. Nat Rev Cancer 11: 588-596.

Langenbacher AD, Huang J, Chen Y, Chen JN. 2012. Sodium pump activity in the yolk syncytial layer regulates zebrafish heart tube morphogenesis. Dev Biol 362: 263-270.

Lee J, Platt KA, Censullo P, Ruiz i Altaba A. 1997. Glil is a target of Sonic hedgehog that induces ventral neural tube development. Development 124: 2537-2552.
Lewis KE, Currie PD, Roy S, Schauerte H, Haffter P, Ingham PW. 1999. Control of muscle cell-type specification in the zebrafish embryo by Hedgehog signalling. Dev Biol 216: 469-480.

Li J, Wang C, Pan Y, Bai Z, Wang B 2011. Increased proteolytic processing of full-length Gli2 transcription factor reduces the hedgehog pathway activity in vivo. Dev Dyn 240: 766-774.

Liem KF Jr, He M, Ocbina PJ, Anderson KV. 2009. Mouse Kif7/ Costal2 is a cilia-associated protein that regulates Sonic hedgehog signaling. Proc Natl Acad Sci 106: 13377-13382.

Lin C, Chen MH, Yao E, Song H, Gacayan R, Hui CC, Chuang PT. 2014. Differential regulation of Gli proteins by Sufu in the lung affects PDGF signaling and myofibroblast development. Dev Biol 392: 324-333.

Liu J, Heydeck W, Zeng H, Liu A. 2012. Dual function of suppressor of fused in Hh pathway activation and mouse spinal cord patterning. Dev Biol 362: 141-153.

Marino S, Nusse R. 2007. Mutants in the mouse NuRD/Mi2 component P66 $\alpha$ are embryonic lethal. PLOS ONE 2: e519.

Matise MP, Epstein DJ, Park HL, Platt KA, Joyner AL. 1998. Gli2 is required for induction of floor plate and adjacent cells, but not most ventral neurons in the mouse central nervous system. Development 125: 2759-2770.

Merchant M, Vajdos FF, Ultsch M, Maun HR, Wendt U, Cannon J, Desmarais W, Lazarus RA, de Vos AM, de Sauvage FJ. 2004. Suppressor of fused regulates Gli activity through a dual binding mechanism. Mol Cell Biol 24: 8627-8641.

Molenaar $M$, van de Wetering $M$, Oosterwegel $M$, PetersonMaduro J, Godsave S, Korinek V, Roose J, Destree O, Clevers H. 1996. XTcf-3 transcription factor mediates $\beta$-catenininduced axis formation in Xenopus embryos. Cell 86: 391399.

Mukhopadhyay S, Rohatgi R. 2014. G-protein-coupled receptors, Hedgehog signaling and primary cilia. Semin Cell Dev Biol 33: 63-72.

Murone M, Luoh SM, Stone D, Li W, Gurney A, Armanini M, Grey C, Rosenthal A, de Sauvage FJ. 2000. Gli regulation by the opposing activities of fused and suppressor of fused. Nat Cell Biol 2: 310-312.

Myers BR, Sever N, Chong YC, Kim J, Belani JD, Rychnovsky S, Bazan JF, Beachy PA. 2013. Hedgehog pathway modulation by multiple lipid binding sites on the smoothened effector of signal response. Dev Cell 26: 346-357.

Nachtergaele S, Whalen DM, Mydock LK, Zhao Z, Malinauskas T, Krishnan K, Ingham PW, Covey DF, Siebold C, Rohatgi R. 2013. Structure and function of the Smoothened extracellular domain in vertebrate Hedgehog signaling. eLife 2: e01340.

Nachury MV. 2014. How do cilia organize signalling cascades? Philos Trans R Soc Lond B Biol Sci doi: 10.1098/ rstb.2013.0456.

Neumann CJ, Grandel H, Gaffield W, Schulte-Merker S, NussleinVolhard C. 1999. Transient establishment of anteroposterior polarity in the zebrafish pectoral fin bud in the absence of sonic hedgehog activity. Development 126: 4817-4826.

Niewiadomski P, Kong JH, Ahrends R, Ma Y, Humke EW, Khan S, Teruel MN, Novitch BG, Rohatgi R. 2014. Gli protein activity is controlled by multisite phosphorylation in vertebrate Hedgehog signaling. Cell Reports 6: 168-181.

Nozawa YI, Lin C, Chuang PT. 2013. Hedgehog signaling from the primary cilium to the nucleus: an emerging picture of ciliary localization, trafficking and transduction. Curr Opin Genet Dev 23: 429-437.

Oh EC, Katsanis N. 2012. Cilia in vertebrate development and disease. Development 139: 443-448.

Ohlmeyer JT, Kalderon D. 1998. Hedgehog stimulates maturation of Cubitus interruptus into a labile transcriptional activator. Nature 396: 749-753. 
Pan Y, Bai CB, Joyner AL, Wang B. 2006. Sonic hedgehog signaling regulates Gli2 transcriptional activity by suppressing its processing and degradation. Mol Cell Biol 26: 3365-3377.

Pearse RV 2nd, Collier LS, Scott MP, Tabin CJ. 1999. Vertebrate homologs of Drosophila suppressor of fused interact with the gli family of transcriptional regulators. Dev Biol 212: 323336.

Petrova R, Joyner AL. 2014. Roles for Hedgehog signaling in adult organ homeostasis and repair. Development 141: 34453457.

Rabinowitz AH, Vokes SA. 2012. Integration of the transcriptional networks regulating limb morphogenesis. Dev Biol 368: 165-180.

Reynolds A, Leake D, Boese Q, Scaringe S, Marshall WS, Khvorova A. 2004. Rational siRNA design for RNA interference. Nature Biotechnol 22: 326-330.

Robbins DJ, Fei DL, Riobo NA. 2012. The Hedgehog signal transduction network. Sci Signal 5: re6.

Rohatgi R, Milenkovic L, Scott MP. 2007. Patched1 regulates hedgehog signaling at the primary cilium. Science 317: 372376.

Ryan KE, Chiang C. 2012. Hedgehog secretion and signal transduction in vertebrates. J Biol Chem 287: 17905-17913.

Santos N, Reiter JF. 2014. A central region of Gli2 regulates its localization to the primary cilium and transcriptional activity. I Cell Sci 127: 1500-1510.

Sasaki H, Hui C, Nakafuku M, Kondoh H. 1997. A binding site for Gli proteins is essential for HNF-3 $\beta$ floor plate enhancer activity in transgenics and can respond to Shh in vitro. Development 124: 1313-1322.

Scales SJ, de Sauvage FJ. 2009. Mechanisms of Hedgehog pathway activation in cancer and implications for therapy. Trends Pharmacol Sci 30: 303-312.

Schmittgen TD, Livak KJ. 2008. Analyzing real-time PCR data by the comparative $\mathrm{C}(\mathrm{T})$ method. Nature Protoc 3: 11011108.

Schulte-Merker S, Stainier DY. 2014. Out with the old, in with the new: reassessing morpholino knockdowns in light of genome editing technology. Development 141: 3103-3104.

Stone DM, Murone M, Luoh S, Ye W, Armanini MP, Gurney A, Phillips H, Brush J, Goddard A, de Sauvage FJ, et al. 1999. Characterization of the human suppressor of fused, a negative regulator of the zinc-finger transcription factor Gli. J Cell Sci 112: 4437-4448.

Svard J, Heby-Henricson K, Persson-Lek M, Rozell B, Lauth M, Bergstrom A, Ericson J, Toftgard R, Teglund S. 2006. Genetic elimination of Suppressor of fused reveals an essential repressor function in the mammalian Hedgehog signaling pathway. Dev Cell 10: 187-197.

Taira T, Maeda J, Onishi T, Kitaura H, Yoshida S, Kato H, Ikeda M, Tamai K, Iguchi-Ariga SM, Ariga H. 1998. AMY-1, a novel C-MYC binding protein that stimulates transcription activity of C-MYC. Genes Cells 3: 549-565.

Tukachinsky H, Lopez LV, Salic A. 2010. A mechanism for vertebrate Hedgehog signaling: recruitment to cilia and dissociation of SuFu-Gli protein complexes. J Cell Biol 191: 415428.

Tuson M, He M, Anderson KV. 2011. Protein kinase A acts at the basal body of the primary cilium to prevent Gli2 activation and ventralization of the mouse neural tube. Development 138: 4921-4930.

Wang B, Fallon JF, Beachy PA. 2000a. Hedgehog-regulated processing of Gli3 produces an anterior/posterior repressor gradient in the developing vertebrate limb. Cell 100: 423-434.

Wang G, Amanai K, Wang B, Jiang J. 2000b. Interactions with Costal2 and suppressor of fused regulate nuclear transloca- tion and activity of Cubitus interruptus. Genes Dev 14: 2893-2905.

Wang Y, McMahon AP, Allen BL. 2007. Shifting paradigms in Hedgehog signaling. Curr Opin Cell Biol 19: 159-165.

Wang C, Pan Y, Wang B. 2010. Suppressor of fused and Spop regulate the stability, processing and function of Gli2 and Gli3 full-length activators but not their repressors. Development 137: 2001-2009.

Wen X, Lai CK, Evangelista M, Hongo JA, de Sauvage FJ, Scales SJ. 2010. Kinetics of hedgehog-dependent full-length Gli3 accumulation in primary cilia and subsequent degradation. Mol Cell Biol 30: 1910-1922.

Wilson CW, Chuang PT. 2010. Mechanism and evolution of cytosolic Hedgehog signal transduction. Development 137: 2079-2094.

Wong SY, Reiter JF. 2008. The primary cilium at the crossroads of mammalian hedgehog signaling. Curr Top Dev Biol 85: 225-260.

Zeng H, Jia J, Liu A. 2010. Coordinated translocation of mammalian Gli proteins and suppressor of fused to the primary cilium. PLOS ONE 5: e15900.

Zhang Y, Fu L, Qi X, Zhang Z, Xia Y, Jia J, Jiang J, Zhao Y, Wu G. 2013. Structural insight into the mutual recognition and regulation between Suppressor of Fused and Gli/Ci. Nat Commun 4: 2608. 


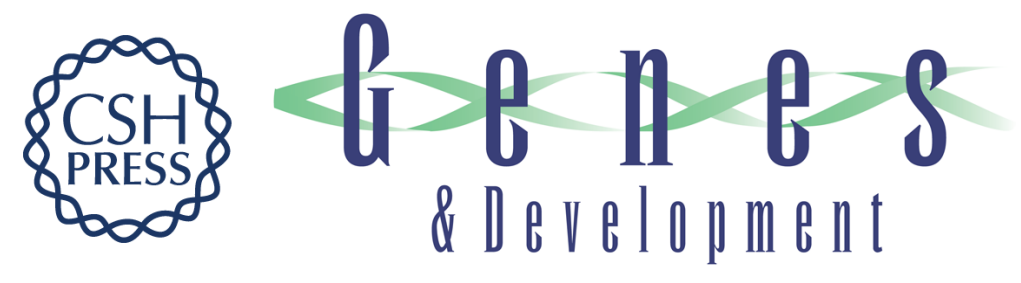

\section{Regulation of Sufu activity by $p 66 \beta$ and Mycbp provides new insight into vertebrate Hedgehog signaling}

Chuwen Lin, Erica Yao, Kevin Wang, et al.

Genes Dev. 2014, 28:

Access the most recent version at doi:10.1101/gad.249425.114

\section{Supplemental http://genesdev.cshlp.org/content/suppl/2014/11/12/28.22.2547.DC1 \\ Material}

References This article cites 102 articles, 37 of which can be accessed free at:

http://genesdev.cshlp.org/content/28/22/2547.full.html\#ref-list-1

Creative This article is distributed exclusively by Cold Spring Harbor Laboratory Press for the first

Commons six months after the full-issue publication date (see

License http://genesdev.cshlp.org/site/misc/terms.xhtml). After six months, it is available under a Creative Commons License (Attribution-NonCommercial 4.0 International), as described at http://creativecommons.org/licenses/by-nc/4.0/.

Email Alerting Receive free email alerts when new articles cite this article - sign up in the box at the top Service right corner of the article or click here.

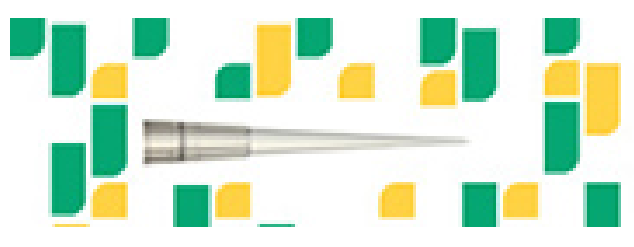

Focused on your science. 